\title{
Chapter
}

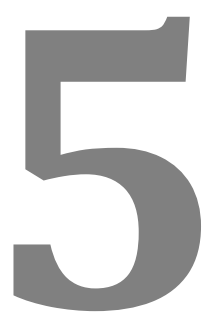

\section{NMR SPECTROSCOPY FOR STUDYING INTERACTIONS OF BIOACTIVE MOLECULES}

Predrag Novak and Tomislav Jednačak

\section{Contents}

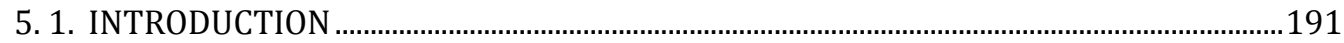

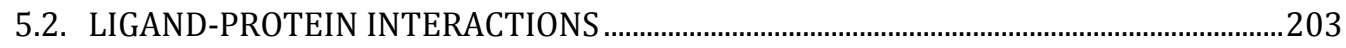

5.3. LIGAND-DNA, LIGAND-RNA AND LIGAND MEMBRANE INTERACTIONS ....................209

5.4. STRUCTURAL ANALYSIS OF LIGANDS...............................................................................214

5.5. STRUCTURAL ANALYSIS OF PEPTIDES AND PROTEINS...................................................215

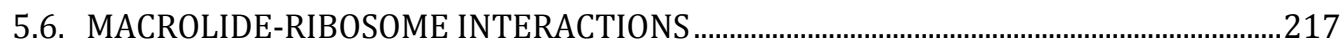

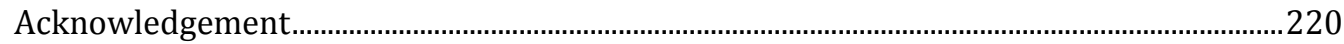

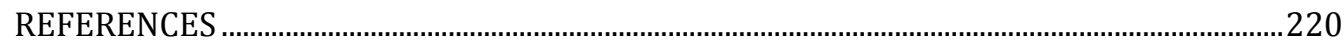





\subsection{INTRODUCTION}

One of the main prerequisites for the successful design of bioactive molecules and drugs is the elucidation of the three-dimensional structure of small molecular ligands, receptors and their complexes. Key steps in this process involve the identification of structural elements and groups responsible for bioactivity. On the other hand, our ability to design novel drug candidates simply from high-resolution structures of biomolecules is still limited. A deeper understanding of molecular mechanisms and dynamics involved in the interaction of ligands with macromolecules is of crucial importance. There are many techniques for probing ligand-receptor interactions, such as equilibrium dialysis, fluorescence spectroscopy, capillary electrophoresis, ultrafiltration, etc. Generally, these techniques require time-consuming separation steps which might influence binding equilibria or analyte derivatisation steps which may change ligand activity. NMR spectroscopy is a very useful method for the characterisation of ligand-receptor interactions since it can provide a wealth of information without destroying the sample. In this review, a description of the main NMR techniques to study the interactions of bioactive molecules with their biological receptors is given and the principal advantages and disadvantages are discussed.

Today, NMR spectroscopy is one of the most powerful and valuable methods in modern drug discovery and development. There is a plethora of one- and multidimensional techniques developed in recent years to study the structures of biologically important molecules such as proteins and nucleic acids and their interactions with other macromolecules or small molecules, for example. Many sequences have been proposed to screen for small molecular binders in the early stages of the drug discovery process. Drug discovery usually includes several critical stages, i.e. hit and lead generation, lead optimisation and preclinical development. Although knowledge of the 3D structures of ligand-receptor complexes might accelerate to a certain extent the search for molecules with higher activity, optimisation of physicochemical properties which dictate in vivo potency and efficacy play an important role as well. These properties include solubility, absorption, distribution, metabolism, excretion and toxicity, known as ADMET.

Initially, the main contribution of NMR spectroscopy to drug design included the determination of the three-dimensional structure of biological targets, mostly 
proteins, and many efforts have been made by biological NMR groups to develop different two- and multi-dimensional techniques for that purpose. The first protein structures determined by NMR were published more than 25 years ago $[1,2]$. Although knowledge of protein structure is certainly an important factor for the drug discovery process, the predictions at that time regarding the impact of NMR were overly optimistic, primarily due to the fact that the size and solubility of proteins are still the biggest limiting factor for successful NMR analysis. Today, protein structure determination by NMR spectroscopy usually includes isotopic labelling of the protein over-expressed in genetically modified bacteria [3]. Hence, the availability of ${ }^{13} \mathrm{C},{ }^{15} \mathrm{~N}$ and ${ }^{2} \mathrm{H}$ isotopically enriched proteins combined with numerous multidimensional NMR experiments enable sequential resonance assignment and solution state structure determination of proteins or proteinligand complexes up to $82 \mathrm{kDa}$ in size [4]. Recently developed sequences which reduce the transverse relaxation rates such as transverse relaxation optimized spectroscopy (TROSY) [5-7] have enabled the recording of high-resolution NMR spectra of macromolecules with masses up to $100 \mathrm{kDa}$ and beyond. However, even for smaller proteins with good resonance dispersion and narrow linewidths, the process of structure determination can be very time consuming. Recent developments in membrane protein NMR studies have revived the role of structural NMR in pharmaceutical research [8]. More recently, new solid-state NMR techniques have emerged as a powerful tool to study proteins that were not accessible to solution state measurements. This has made possible studies of integral membrane proteins, protein aggregates and fibrous proteins, regardless of the solid state characteristics of the studied system, which is one of the main prerequisites for X-ray diffraction analysis where crystalline samples are needed. More recent information on protein structure analysis can be found in the related literature [9-13]. In modern drug discovery, NMR provides much more than macromolecular 3D structure determination since it has found widespread use in hit and lead generation, metabonomics and complex mixture analysis. Excellent discussions of the principles and applications of NMR techniques in metabonomics and mixture analysis can be found in a number of books and articles [14-21].

Apart from the structural information on the macromolecular target in the process of drug design, it is crucial to understand the principles of how a particular biological receptor interacts with a drug-like molecule. This is one of the prerequisites for the discovery of high affinity ligands for biologically important protein targets. Ligand-receptor interactions by using NMR might also include the elucidation of free and bound ligand conformations, the mobility of the ligand, receptor and their complex, determination of binding constants, determination of the reactive groups responsible for binding and also provide information on the orientation or immersion depth of the ligand in its target.

The binding of a ligand molecule to its receptor in its simplest form follows the bimolecular association reaction with second-order kinetics according to Equation 5.1: 


$$
\mathrm{R}+\mathrm{L} \underset{k_{\text {off }}}{\stackrel{k_{\text {on }}}{\rightleftarrows}} \mathrm{RL}
$$

where $k_{\text {on }}$ and $k_{\text {off }}$ are the on (association) and off (dissociation) rate constants.

The dissociation constant $K_{\mathrm{D}}$ can then be expressed by Equation 5.2:

$$
K_{d}=\frac{k_{\mathrm{off}}}{k_{\mathrm{on}}}=\frac{[\mathrm{R}][\mathrm{L}]}{[\mathrm{RL}]}
$$

As already mentioned, there have been many NMR sequences developed in the last two decades that can provide useful information on the interactions between biological targets and small molecules which can be used to screen for potential binders [22-28]. These can be divided into two main groups: ligand-based and receptor-based techniques. Theoretically, all NMR parameters are sensitive to binding processes and can be used to study ligand-receptor interactions. Most frequently used are those that are easily obtained and with highest sensitivity and include chemical shifts, translational diffusion coefficients, relaxation times and the nuclear Overhauser effect (NOE). Some of these parameters are simply used to indicate if binding occurs, while others provide information on the location of ligand binding sites and surface mapping features and determine binding constants and changes in the mobility of ligand-receptor complexes. NMR screening for biologically active molecules includes the identification of small molecule ligands for macromolecular targets by the observation of a change in an NMR parameter upon interaction. The parameters that are usually used to study interactions between ligand and their biological receptors are given in Figure 5.1.
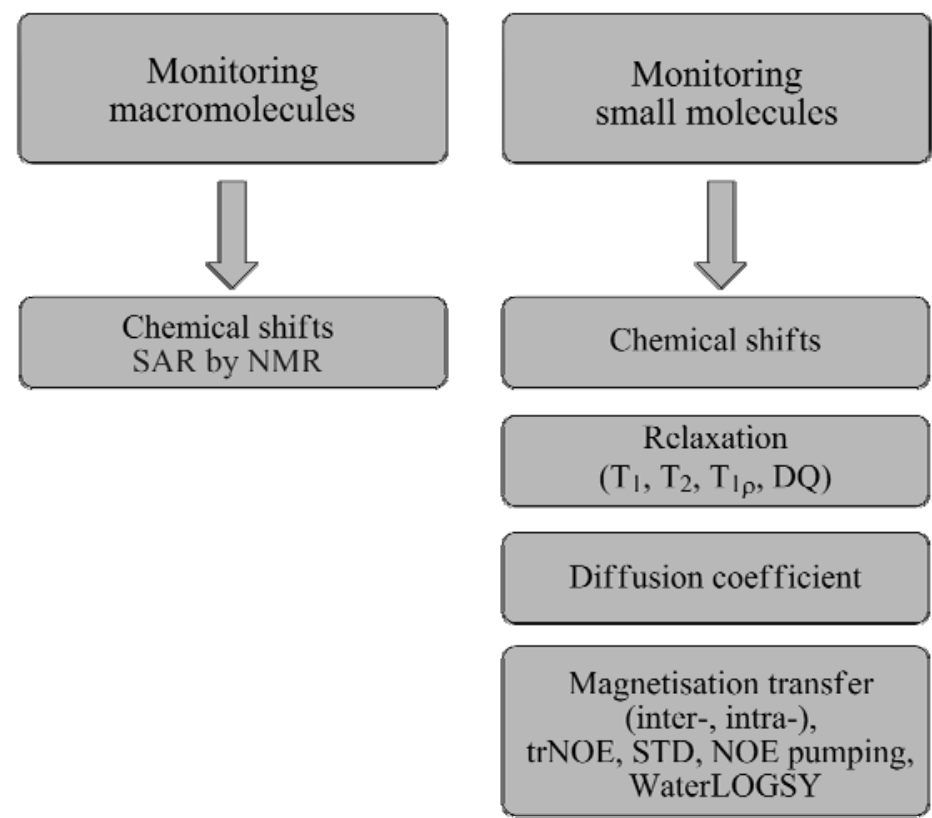

Figure 5.1. NMR parameters used for studying ligand-receptor interactions 
Both approaches have advantages and disadvantages. The receptor-based methods do not rely on fast exchange and therefore allow for the characterisation of both high and low affinity binders. They can also easily distinguish between specific and non-specific binding. The main prerequisite for this approach is $a$ priori knowledge of the 3D structure of the protein obtained either from NMR or $\mathrm{X}$-ray diffraction analysis. Monitoring changes in NMR parameters (usually chemical shifts) identifies not only binding of molecules, but also localises their binding sites. Some major drawbacks of this approach include the size of the target protein which prevents complete resonance assignment and structure elucidation as well as isotopic enrichments ${ }^{13} \mathrm{C},{ }^{15} \mathrm{~N},{ }^{2} \mathrm{H}$ etc.) necessary for structural analysis. One of the main disadvantages of receptor-based techniques is the overexpression and purification of milligram quantities of a soluble protein, since in drug discovery, targets are selected on the basis of their therapeutic potential and not their compliance with NMR. For ligand-based methods, the target size is irrelevant and there is no need for isotopic labelling. The receptor concentration is much smaller, usually in the micromolar range. This approach mostly utilises 1D techniques, which are faster than 2D or higher dimensional techniques needed to solve protein structures. There are also some drawbacks connected to these techniques, such as the inability to a priori localise the binders on the receptor and the requirement for fast exchange between free and bound states. Also, the large excess of ligand molecules used in such experiments might increase the risk of non-specific binding. However, the ligand-based approach has broader applicability today and is frequently used for primary and secondary screening.

An excellent receptor-based method called structure-activity relationship (SAR) by NMR was developed by researchers at Abbott [29-31]. The method is based on perturbations in protein hetero-nuclear single quantum coherence NMR spectra $\left({ }^{1} \mathrm{H}-{ }^{15} \mathrm{~N}\right.$ or $\left.{ }^{1} \mathrm{H}-13 \mathrm{C}-\mathrm{HSQC}\right)$ upon the addition of a compound mixture. It requires ${ }^{15} \mathrm{~N}$ or ${ }^{13} \mathrm{C}$ isotopic enrichment of proteins. In the first step, a library of small molecules is screened to identify those that bind to the target protein. Binding is detected by comparing two-dimensional ${ }^{1} \mathrm{H}-{ }^{15} \mathrm{~N}$ or ${ }^{1} \mathrm{H}-13 \mathrm{C}-\mathrm{HSQC}$ spectra of the protein prior to and after the addition of a ligand or ligand mixture to detect changes in chemical shifts upon binding. The ligands that bind to one or more pockets in spatial proximity are identified, optimised and linked together to produce high-affinity ligands. Since the first application of this method on the FKBP protein, it has been used to discover many high-affinity ligands for other therapeutically relevant proteins.

For experiments that rely on the observation of ligand molecules, the choice of NMR parameters is more diverse (Figure 5.1). The high sensitivity of ligand parameters to binding have enabled a number of valuable techniques to be developed to better understand the binding process at a molecular level and to identify new bioactive compounds [22-28]. Upon binding of a small molecule ligand to its target macromolecule, the chemical shifts of both the ligand and the target resonances are perturbed. Hence, for the detection of whether a ligand binds or not, in the case of well-separated signals it is sufficient to perform simple titration expe- 
riments and observe changes in proton chemical shifts and line-widths. This is shown in Figure 5.2 for binding of the antibiotic erithromycin to the ribosome.

The dissociation constant may also be estimated by nonlinear curve fitting of the chemical shifts vs. concentration. Other methods to identify and characterise ligand-receptor interactions can generally be divided into NOE-based, relaxationbased and diffusion-based methods.

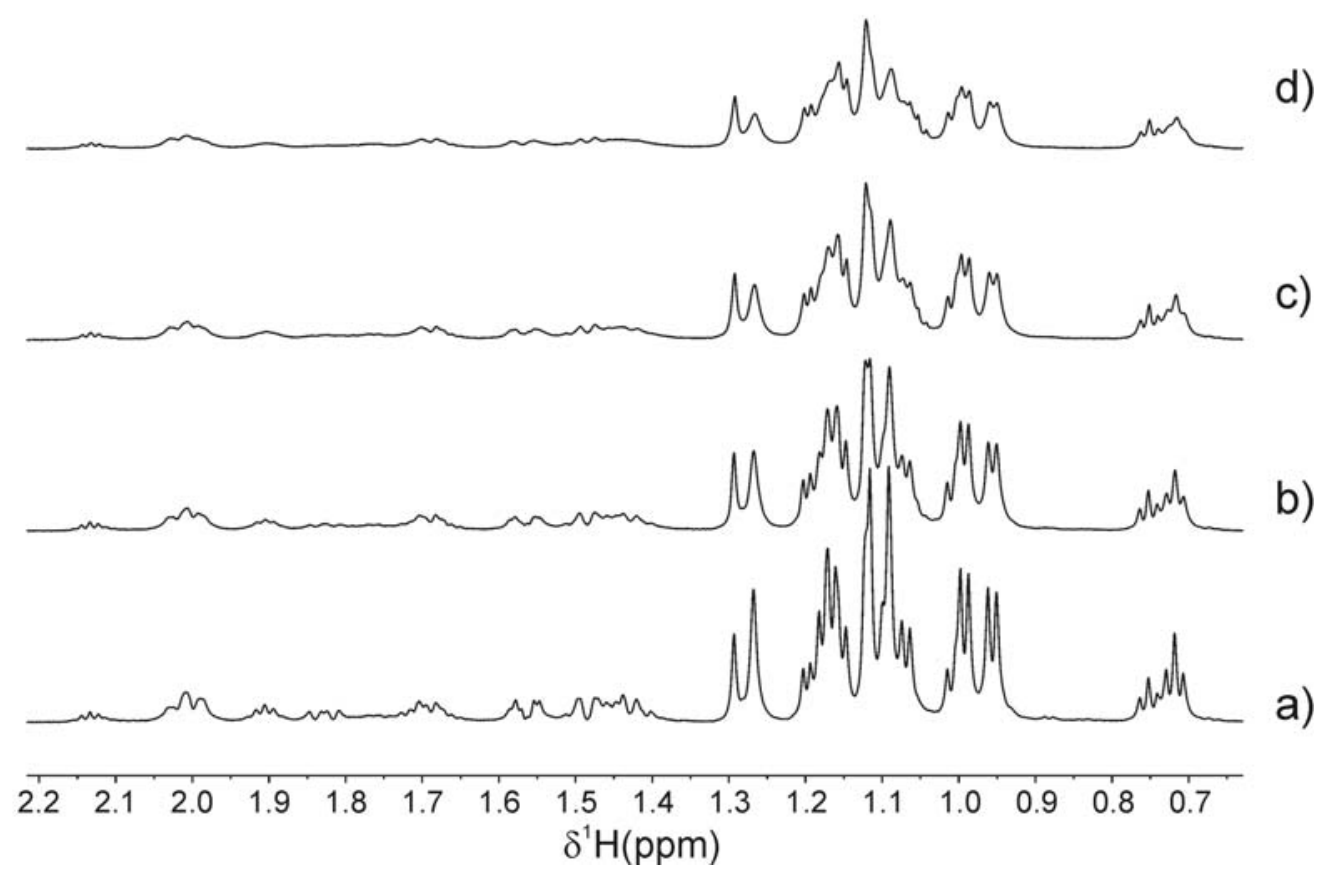

Figure 5.2. Proton spectrum of erithromycin (a) and proton spectra of erythromycin after the incremental addition of E. coli ribosome (lines b, c and d) recorded at $700 \mathrm{MHz}$

A number of NMR techniques based on the NOE effect have been developed recently. It is well known that NOEs are invaluable tools for determining 3D structures of small molecules and biomolecules. Upon binding of the ligand molecule to its biological receptor, the NOEs undergo significant changes leading to the observation of transferred NOEs or transferred nuclear Overhauser enhancements (trNOEs) which stem from different correlation times, $\tau_{c}$, of free and bound molecules. Small molecular ligands have short correlation times and usually give rise to positive NOEs or no NOEs. On the other hand, large macromolecules exhibit strong negative NOEs. This is depicted in Figure 5.3. If a small molecule binds, it behaves like a macromolecule and shows strong negative trNOEs, providing a fast exchange regime between the bound and free states.

This is demonstrated in Figure 5.4 for binding of the macrolide antibiotic azithromycin to ribosomes isolated from E. coli. It can be seen that the 2D NOESY spectrum of azithromycin in buffered solution displays only a few positive NOE 


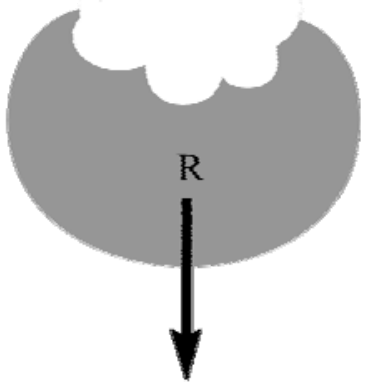

slow Brownian motion:

fast relaxation; negative NOE; slow diffusion

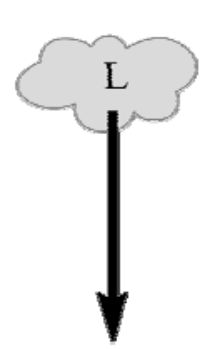

fast Brownian motion:

slow relaxation; positive NOE; fast diffusion
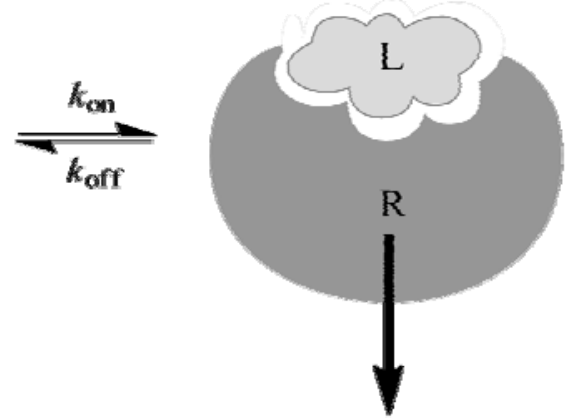

motional properties of $\mathbf{L}$ similar to those of $\mathbf{R}$

Figure 5.3. Properties of the ligand, receptor and their complex

cross-peaks (all others vanish since $\tau_{\mathrm{c}} \omega_{0} \approx 1, M w=774$ ). However, after the addition of $70 \mathrm{~S} E$. coli ribosomes, the observed trNOEs are all negative with higher intensities, indicating interactions between azithromycin and ribosomes and the formation of a complex. In practise, these experiments are performed with high excess of the ligand (from ten to hundred-fold excess) so that signals of the receptor molecules are not visible in the spectrum and no filtering techniques are necessary. Furthermore, the analysis of trNOEs provides information on the bound conformation. In addition, one can also observe intermolecular trNOEs between the ligand and receptor protein which allows for the determination of the orientation of bound ligands in protein binding pockets [32,33].

Another very popular and fast method that relies on the NOE effect is saturation transfer difference NMR spectroscopy (STD-NMR), proposed by B. Mayer and M. Meyer [34,35]. The method has become one of the most frequently used NMR methods to characterise interactions between ligands and receptors. It is based on the transfer of saturation from the receptor to the bound ligand which in turn is moved into solution by exchange and detected. As depicted in Figure 5.5, STD takes the difference of the two experiments. In the first experiment (on-resonance), only receptor resonances are selectively irradiated or saturated using selective pulses. The saturation propagates to all receptor protons via the network of an efficient intra-molecular proton-proton cross-relaxation process known as spin diffusion. As seen in Figure 5.5, compounds that bind to the receptor become saturated as well via inter-molecular proton-proton cross-relaxation. The saturation is then carried into solution by the exchange process and detected. Subsequently, a reference spectrum is recorded with the irradiation frequency set far from any signal giving rise to an off-resonance spectrum. The subtraction of the on-resonance spectrum from the off-resonance spectrum gives rise to a difference spectrum, showing only resonances that have experienced saturation. 


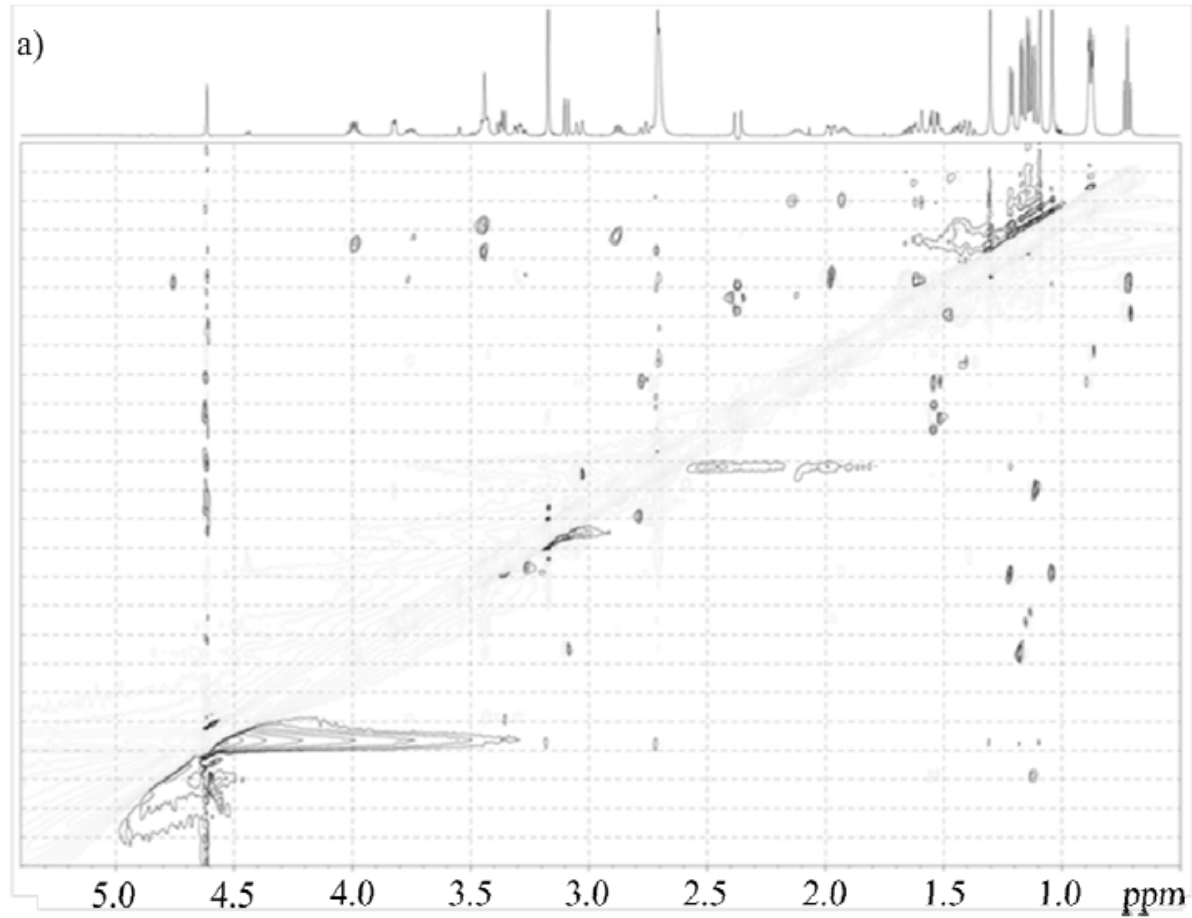

b)
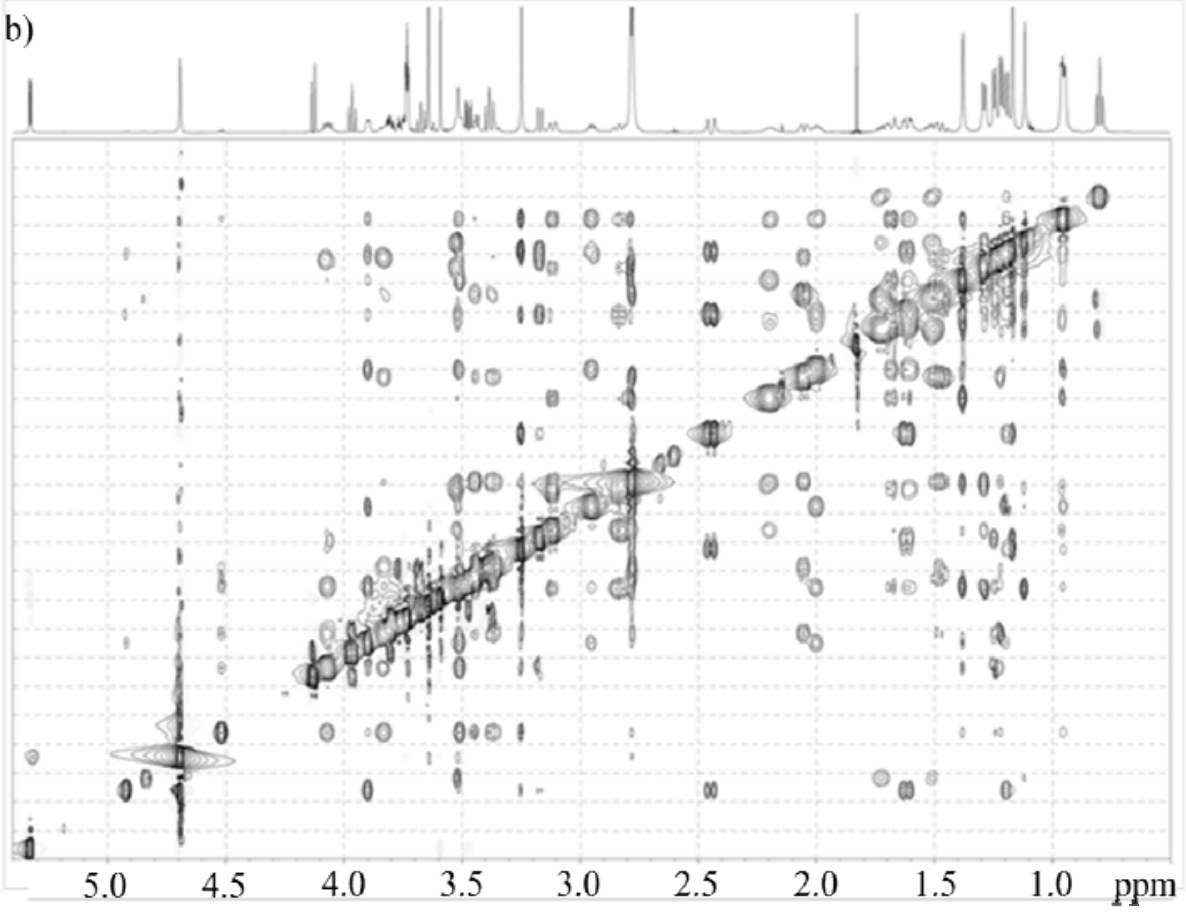

Figure 5.4. (a) NOESY spectrum of azithromycin in TRIS buffer and (b) TrNOESY spectrum of azithromycin in TRIS buffer after the addition of 70S E. coli ribosomes 
The degree of ligand saturation depends on the residence time of the ligand in the receptor binding pocket. When it dissociates from the receptor, the saturation is transferred to the solution where the free ligand gives rise to signals with narrow line widths. There are several advantages of the STD method.

First, STD has no limitation with respect to the size of the receptor and is ideal for large receptor proteins and even whole ribosomes (see Section 5.6). Second, it

a)
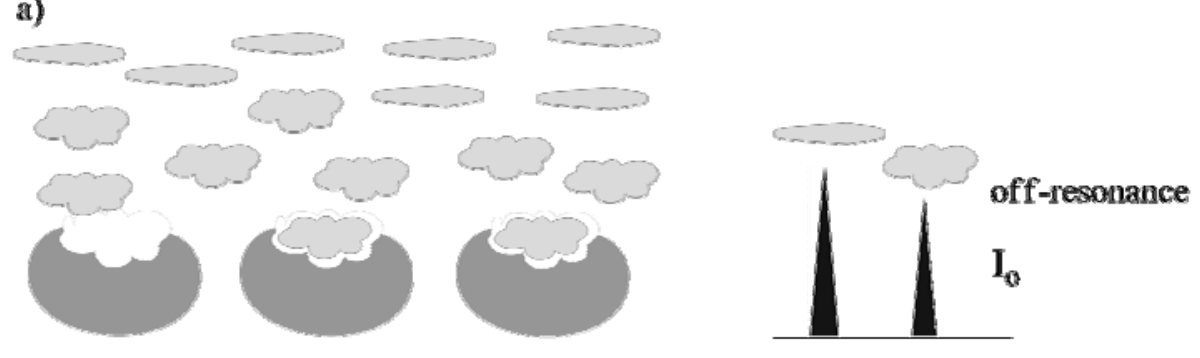

b)

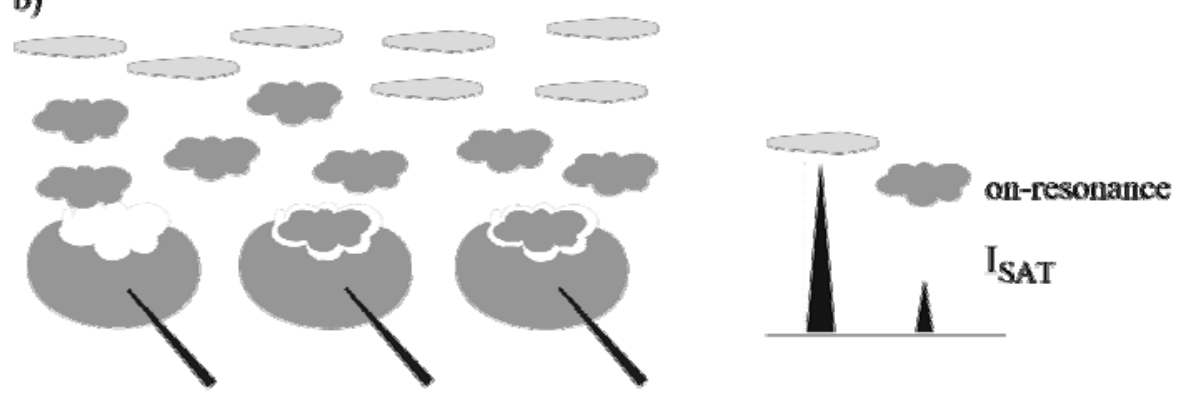

c)
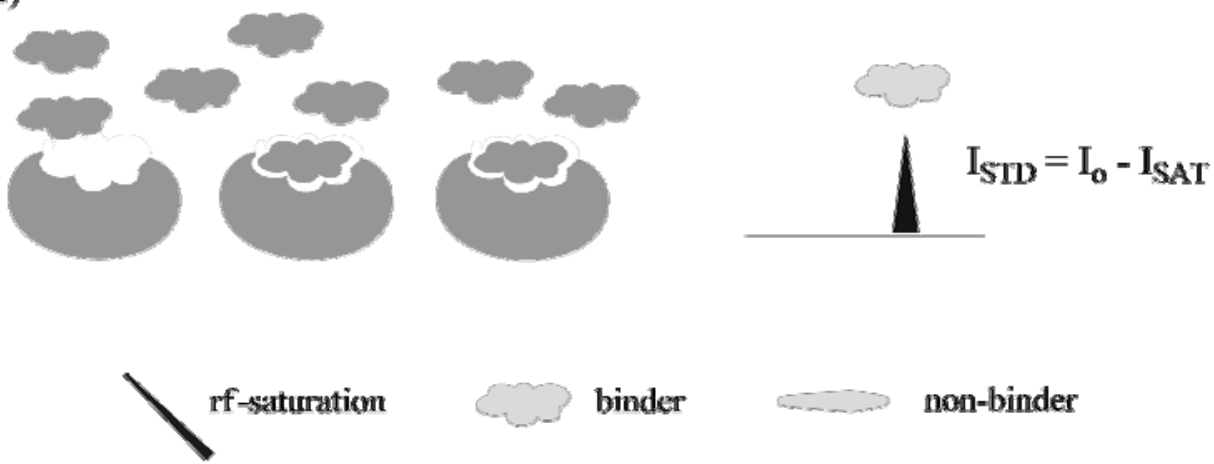

binder

non-binder

Figure 5.5. Schematic representation of an STD experiment: (a) the off-resonance or reference experiment involves rf-irradiation off-resonance from ligand and receptor protons giving intensity $\mathrm{I}_{0}$, (b) the on-resonance experiment applies rf-irradiation for selective saturation of receptor resonances producing attenuated signal intensity $I_{\text {SAT }}$ and (c) STD spectrum which gives only resonances of the binding compounds (receptor resonances are usually invisible due to either low concentrations or relaxation filtering) 
requires low amounts of the receptor (usually in the micromolar range) and it benefits from the fact that only bound ligands show signals in the spectrum and therefore is very suitable for screening purposes. A typical one-dimensional STD pulse sequence is shown in Figure 5.6.

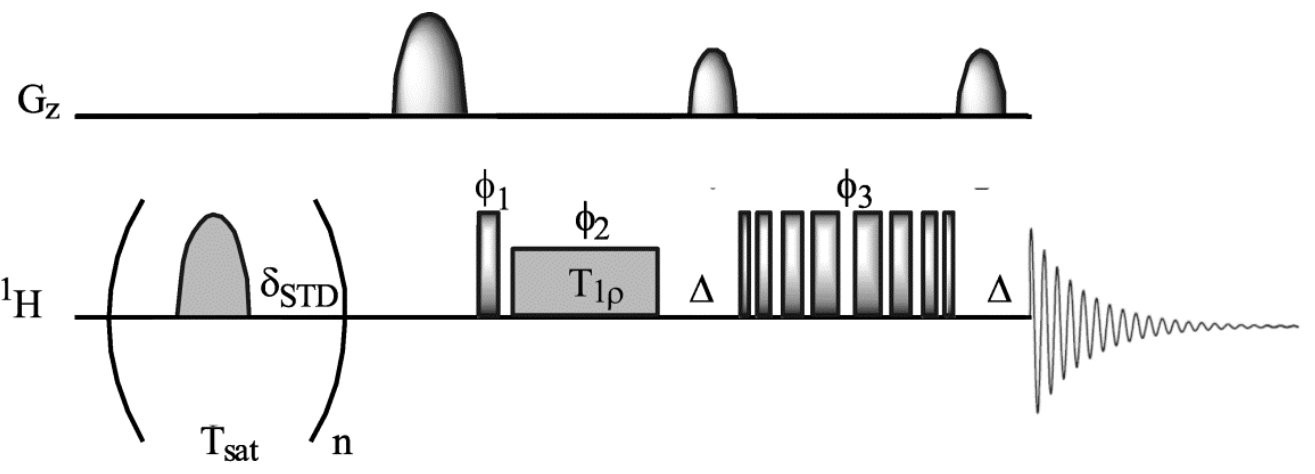

Figure 5.6. STD pulse sequence with a $\mathrm{T}_{1 \rho}$ relaxation filter to suppress unwanted receptor resonances

Furthermore, STD may be combined with other pulse sequences, thus generating experiments such as STD - total correlation spectroscopy (STD-TOCSY) or STDHSQC, for example [36]. STD-NMR spectroscopy can be successfully used to identify groups of atoms in direct contact with the receptor since these experience the highest degree of saturation; this allows for epitope mapping or group epitope mapping (GEM) [35]. Protons closest to the target molecule are saturated to a higher degree, giving stronger STD enhancements. This information can be used to identify structural moieties that are important for binding. The binding constants can also be assessed from STD-NMR spectra by performing competition titration experiments. A variation of STD-NMR spectroscopy is the water ligand-observed gradient spectroscopy (waterLOGSY) method which uses the fact that intermolecular water-ligand NOE will be negative only when the ligand is bound to the protein $[37,38]$. The transfer of magnetisation is from water to receptor to binding ligand. The method utilises either the steady-state NOE experiment with on-resonance saturation applied to the water signal or selective inversion of the water resonance signal in the enhanced protein hydration observed through gradient spectroscopy nuclear Overhauser effect (e-PHOGSY NOE) experiment [39]. Protein-ligand interactions can also be studied by monitoring inter-molecular NOEs using NOE pumping or reverse NOE pumping (RNP) techniques [40]. RNP uses a relaxation filter followed by the NOE mixing element where polarisation is transferred from a binding ligand to the protein, resulting in a spectrum containing only the signals of the binding ligands. Recently, a method called INPHARMA (Interligand Noes for PHARmacophore MApping) methodology was introduced [41]. It is based on the observation of spin-diffusion mediated, trNOE data between two ligands $\left(\mathrm{L}_{1}\right.$ and $\mathrm{L}_{2}$ ) that bind weakly and competitively to a macromolecular receptor (R). As $\mathrm{L}_{1}$ binds to the receptor, the magnetisation is transferred from the ligand to the 
receptor protons during the mixing time of the NOESY experiment. During the same mixing time, $\mathrm{L}_{1}$ dissociates from the receptor and $\mathrm{L}_{2}$ binds, which allows the transfer of magnetisation from the receptor to $\mathrm{L}_{2}$ protons.

The chemical exchange during INPHARMA experiment can be explained by two different models:

a) A three-step model, which includes the state where both ligands and the receptor are found also in free form. The three-step model can be described by:

$$
\mathrm{RL}_{1}+\mathrm{L}_{2} \underset{k_{1 \text { on }}}{\stackrel{k_{1 \text { off }}}{\rightleftarrows}} \mathrm{L}_{1}+\mathrm{R}+\mathrm{L}_{2} \underset{k_{2 \text { off }}}{\stackrel{k_{2 \text { on }}}{\rightleftarrows}} \mathrm{L}_{1}+\mathrm{RL}_{2}
$$

b) A two-step model, where the receptor is never in free form, due to the presence of the two ligands in large excess. This model can be described by:

$$
\mathrm{RL}_{1}+\mathrm{L}_{2} \underset{k_{21}}{\stackrel{k_{12}}{\rightleftarrows}} \mathrm{L}_{1}+\mathrm{RL}_{2}
$$

In contrast to STD and waterLOGSY experiments, the information obtained in the INPHARMA spectrum is highly site-specific. INPHARMA provides rapid determination of the relative orientation of different chemical structures in the macromolecule binding pocket. INPHARMA methodology was successfully applied to a mixture of baccatin III $\left(\mathrm{L}_{1}\right)$ and epothilone $A\left(\mathrm{~L}_{2}\right)$ in the presence of tubulin (R). The results showed that the intermolecular NOEs between epothilone $A$ and baccatin III were mediated by the protons in tubulin.

Binding of the ligand to the target will have a dramatic effect to its relaxation properties. Free small molecule ligands are characterised by small longitudinal and transverse relaxation rates, $\left(R_{1}=1 / T_{1}\right.$ and $\left.R_{2}=1 / T_{2}\right)$. When bound, ligands share relaxation properties of the receptors with large relaxation rates. Therefore, binding will cause significant line-broadening of ligand resonances. A comparison of transverse relaxation times in the presence and absence of the receptor is very sensitive probe of binding due to its almost direct dependence on the overall molecular correlation time. $T_{2}$ can be determined by using a simple Carr-PurcellMeiboom-Gill (CPMG) experiment [42,43] or, alternatively, one can use $T_{1}$ determined from rotating frame Overhauser effect spectroscopy (ROESY)-type experiments to detect binding. A variation of the relaxation-based approach is the method called SLAPSTIC (Spin Labels Attached to Protein Side chains as a Tool to identify Interacting Compounds) [44,45] which allows for the screening of primary binding sites by covalently attaching spin labels to protein targets. The use of paramagnetic relaxation enhancement (PRE) experiments could provide further structural information on the orientation or immersion depth of the ligand in its target. Upon the addition of an inert paramagnetic agent to the solvent, relaxation enhancements of ligand nuclei depend on the insertion depth in the target. If the ligand is in fast exchange between the free and bound state, 
solvent PREs are partly transferred to the free ligand where they can be observed with high resolution and without any size limitation of the receptor [46-49]. Information regarding the orientation of the ligand with respect to the receptor can be determined and further used for guiding docking calculations, provided that the three-dimensional atomic resolution structure of the receptor is available. Identifying parts of a drug which are buried deepest in the receptor can help with directing chemical modifications of the ligand to design better inhibitors.

Experiments based on translational diffusion can also be used to probe interactions of ligands with a target. The diffusion-editing techniques are based on the fact that, upon binding, the hydrodynamic radius $r_{\mathrm{H}}$ of the ligand increases substantially, leading to a decrease in the diffusion rate. The translational diffusion coefficients can be obtained according to Stokes-Einstein equation:

$$
D=k T / 6 \eta \pi r_{\mathrm{H}}
$$

where $k$ represents the Boltzmann constant, $T$ is the temperature, $\eta$ is the solvent viscosity and $r_{\mathrm{H}}$ is hydrodynamic radius. From Equation 5.5, it is clear that a small ligand molecule with a small hydrodynamic radius will have larger diffusion coefficient with respect to larger molecules such as proteins. Hence, if a ligand binds to the receptor, its diffusion coefficient will decrease. A variety of NMR pulse sequences have been developed to investigate diffusion coefficients in solution [50]. Diffusion-editing has been successfully used for the characterisation of interactions between small and intermediate sizes proteins. With the development of reliable gradient technology, many NMR pulse schemes using gradients have been proposed to determine diffusion coefficients such as bipolar pulsed field gradient longitudinal eddy-current delay (bPPFGLED), depicted in Figure 5.7.

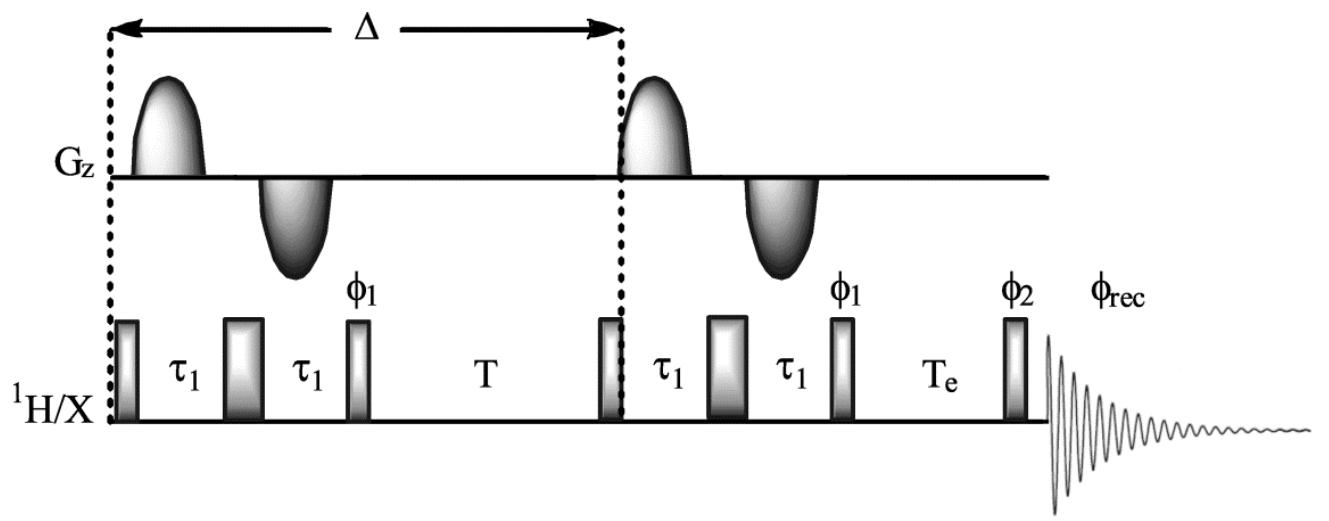

Figure 5.7. The bpPFGLED pulse sequence.

The signal intensity during the diffusion experiment will decay exponentially according to Equation 5.6:

$$
I=I_{0} \exp \left[-D(\gamma \delta G)^{2}(\Delta-\delta / 3)\right]
$$


where $I_{0}$ is the signal intensity in the absence of field gradients, $D$ is the diffusion coefficient, $\gamma$ is the gyromagnetic ratio, $\delta$ is gradient length, $G$ is gradient strength and $\Delta$ is the diffusion time. Diffusion coefficients can be determined from the slope of the linear dependence of the natural logarithm of both sides of the Equation 5.6. The experimental parameters can be optimised to suppress the signals of non-binding compounds and to detect the resonances of bound ligands.

Figure 5.8 shows the results from an experiment performed on a mixture of three compounds in the presence of porcine pancreatic elastase. It can be seen that only the signals of the compound that binds, e.g. phenylphosphonic acid, appears in the spectrum of the mixture, while signals of the other non-binding compounds are not present.

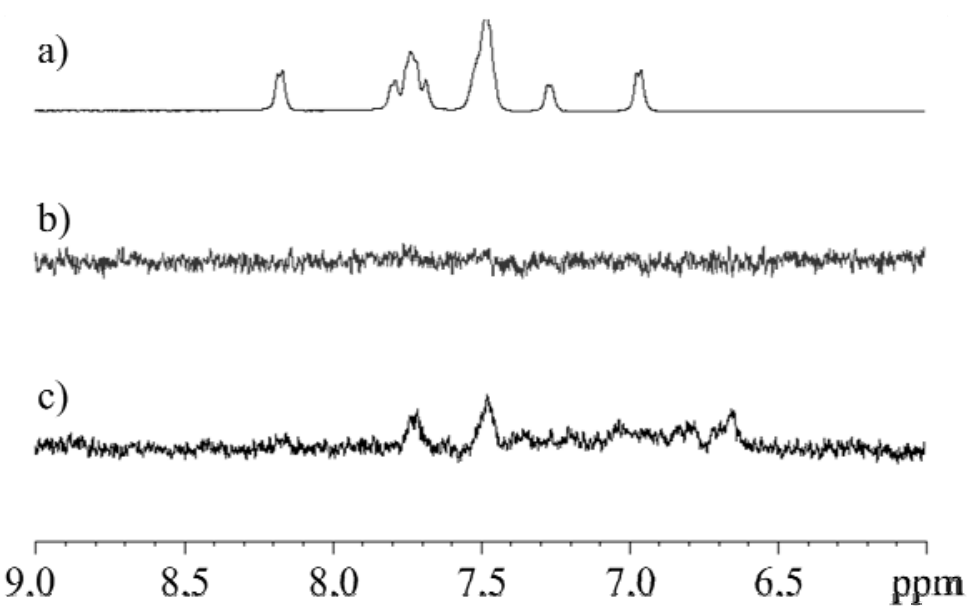

Figure 5.8. The bpPFGLED spectra of a mixture of phenylphosphonic acid and two small organic compounds at (a) low gradient strength, (b) high gradient strength and

(c) after the addition of porcine pancreatic elastase at high gradient strength.

Diffusion-based methods can further be used to provide knowledge on which parts of the ligand are involved in the binding surface and to determine the binding epitopes. A key advantage of diffusion epitope mapping over the aforementioned GEM approach is that it bypasses problems of longitudinal relaxation, which in some cases might lead to erroneous STD enhancements in GEM experiments. The main disadvantage of diffusion experiments is their lower sensitivity compared to relaxation-based or NOE-based approaches and some limitations exist regarding the size of the receptor.

Although very powerful and efficient, ligand-based NMR methods also have some limitations. Most of these methods report cases of weak to medium affinity binding. Usually, these experiments are performed with a large excess of ligands which may lead to non-specific binding. Other limitations include molecules with low water solubility, those that bind with high affinity to the receptor and molecules that have small off-rate constants and hence are not detected. In such cases, competition experiments are very useful to monitor ligand-receptor 
interactions, thus avoiding many drawbacks connected with the direct ligandbased methodologies [51-54]. Competition ligand-based methods are robust and reliable tools for providing valuable information on specific binding and could serve as quantitative measures of the ligand affinity for the receptor. A competition method that combines waterLOGSY and transverse relaxation has recently been proposed as a sensitive method for fragment screening [55].

The NMR methods discussed above will continue to play significant roles in the process of modern drug discovery, including hit generation, lead generation and optimisation phases, thus increasing the chances of success to produce drug candidates with high potency, reduced toxic effects and desired physicochemical properties.

In the next section, recent applications of the NMR techniques discussed above to study ligand-receptor interactions are reviewed.

\subsection{LIGAND-PROTEIN INTERACTIONS}

Ligand-protein interactions can be monitored by various experimental methods. As mentioned above, ligand-based NMR techniques are useful tools for investigations of such interactions.

STD-NMR spectroscopy was used to investigate the binding properties of the caged nucleotides guanosine 5'-O-(3-thiotriphosphate), $P$ 3(S)-(1-(4,5-dimethoxy-2-nitrophenyl)ethyl) ester (DMNPE-GTP- $\gamma$-S) and adenosine 5'-diphosphate, $P^{2}$-(1-(2-nitrophenyl)ethyl) ester (NPE-ADP) to rabbit muscle creatine kinase (RMCK) and human annexin A6 (hAnxA6) [56]. The obtained results indicated the strong binding of caged nucleotides to the investigated proteins. Similarly, Blume et al. [57] used the same technique to study the interaction of the epimerase site of the bifunctional enzyme UDP- $N$-acetylglucosamine-2-epimerase/ $N$-acetylmannosamine kinase with its natural substrate, UDP- $N$-acetylglucosamine (UDP-GlcNAc) and derivatives. STD-NMR allowed for ligand epitope mapping and the determination of ligand binding affinity. The binding epitopes of the uridine moieties of UMP, UDP, UDP-GalNAc and UDP-GlcNAc were similar, suggesting that the binding mode of the UDP moiety was the same in all cases. However, small differences in the binding epitopes of hexopyranose units of UDPGlcNAc and UDP-GalNAc reflected the inability of the enzyme to process UDPGalNAc. The results of STD titrations showed that UDP had the greatest binding affinity to the epimerase site of the enzyme.

Furthermore, a combination of STD-NMR and ${ }^{1} \mathrm{H}-{ }^{15} \mathrm{~N}$ HSQC experiments was used to investigate the binding of 5-aminoimidazole-4-carboxamide-ribonucleoside (AICAR) and guanosine monophosphate (GMP) to histidine triad nucleotide binding protein (HINT1) [58]. The presence of ligand binding was checked using STD-NMR spectroscopy. The ligand binding site was established from ${ }^{1} \mathrm{H}$ and ${ }^{15} \mathrm{~N}$ chemical shift perturbations. The binding of AICAR to HINT1 revealed 
considerable chemical shift perturbation similarity to GMP, indicating that the two ligands probably bind in the same basic location.

Ji et al. [59] applied STD-NMR spectroscopy to map a group epitopes and to measure the dissociation constant of specific interactions between L-tryptophan (Trp) and human serum albumin (HSA). STD spectra recorded in ligand-protein solutions with and without the inhibitor naproxen pointed towards the interaction between Trp and HSA. The STD spectrum in the absence of the inhibitor reflected specific as well as non-specific binding of Trp. However, in the presence of naproxen, the specific sites were occupied by the inhibitor. Hence, the resulting STD spectrum reflected only non-specific contributions to the interaction between Trp and HSA.

Several groups have studied competitive ligand binding using STD-NMR spectroscopy. Sadeghi-Khomami et al. [60] studied the binding of pNP- $\beta$-Dglucose to hydrolytically inactive nucleophile mutants of the family-3 retaining $\beta$ glucosidase DesR in the presence of erythromycin A. The results of competitive STD-NMR experiments indicated that erythromycin A binds to the active site and acts as a competitive glycosidase inhibitor. Similarly, STD-NMR spectroscopy was used to study the interaction between bifunctional galactofuranosyltranferase GlfT2 and two trisaccharide acceptor substrates [61]. The bifunctionality of the enzyme was explored by competition STD experiments and saturation transfer double difference experiments (STDD). The results showed that both acceptor substrates bind competitively at the same site.

STD-NMR spectroscopy provided insight into enzyme-ligand interactions in yeast hexokinase isoform PII under physiological conditions [62]. Competitive STD titration experiments allowed for ranking the ligands according to their binding affinities.

Goncalves et al. [63] investigated the binding of a synthetic Toll-like receptor (TLR4) antagonist to cluster differentiation antigen CD14 by STD-NMR. The results indicated that the binding was principally mediated through the lipid chains of the synthetic compound. These findings strongly suggest that TLR4 activation is inhibited by competitively occupying CD14.

Furthermore, the competition STD method was successfully applied to detect the presence of a competing high-affinity ligand, diazepam, in a compound mixture by the reduction of the STD signals of a low-affinity ligand, $\beta$-site amyloid precursor protein cleaving enzyme 1 [64].

Plesniak et al. [65] characterised the conformation of novobiocin in the pocket of histidine protein kinase EnvZ using STD and trNOE experiments. Thus, STD-NMR spectroscopy identified the novobiocin moieties that interacted with EnvZ. The authors suggested that novobiocin binds to EnvZ in a conformation and orientation similar to its binding to DNA gyrase B.

Similarly, Lee et al. [66] characterised binding inhibitors of Escherichia coli $\beta$ ketoacyl-acyl carrier protein synthase III (ecKAS III) using STD-NMR 
spectroscopy. The results suggested that the antimicrobial compound, 4cyclohexyliminomethyl-benzene-1,3-diol (YKAs3003), was a potent inhibitor of pathogenic KAS III. It was shown that the binding models of YKAs3003 and ecKAS III determined from in silico screening correlated well with the STD results.

According to Houliston et al. [67] STD experiments enable the investigation of antibody-ligand interaction in target ganglio-oligosaccharides added directly to patient sera, without antibody fractionation. Very strong saturation transfer was observed in one-third of glycan resonances upon the addition of gangliooligosaccharides to patient sera; this delineated the region of contact with serum antibodies.

Murata et al. [68] have shown how STD-NMR spectroscopy can be applied to identify binding epitopes and to study the binding of gibberelin $A_{3}$ to the monoclonal antibody 4-B8(8)/E9. STD experiments provided useful information on the hapten-antibody interaction in the solution state. The results obtained by STDNMR were in excellent agreement with the results of crystallographic analysis.

Assadi-Porter et al. [69] demonstrated the use of STD and STDD-NMR experiments for monitoring specific ligand binding to the human taste type 1 receptors 2 and 3 (T1R2 + T1R3). Subtraction of STD spectra recorded in the presence and the absence of sweet receptors yielded two-dimensional ${ }^{1} \mathrm{H}-{ }^{15} \mathrm{~N}$ HSQC STDD spectra containing signals only from specific binding. The results indicated that the mutant receptor (T1R2 + T1R3(D535Q)) binds the ligand brazzein much less tightly than does the wild-type receptor (T1R2+T1R3).

Streiff et al. [70] proposed the application of STD-NMR spectroscopy for anaesthetic binding proteins. The measurements were conducted on a series of protein/anaesthetic solutions. STD spectra provided evidence for $\mathrm{Ca}^{2+}$-dependent halothane binding to calmodulin. Those findings demonstrated the specificity of anaesthetics for a particular protein conformation.

Macnaughtan et al. [71] combined STD-NMR and trNOE experiments to study the conformation of UDP-GlcNAc and $\beta$-D-GlcpNAc- $(1 \rightarrow 2)-\alpha$-D-Manp $(1 \rightarrow 6)-\beta$-D-GlcpO0ctyl, as well as their interaction with $N$-acetylglucosaminyltransferase $\mathrm{V}$. In addition, the relative orientation of the two bound ligands was determined in a paramagnetic relaxation enhancement experiment using a spin-labelled ligand analogue, 5'-diphospho-4-O-2,2,6,6-tetramethylpiperidine-1-oxyl (UDP-TEMPO).

Furthermore, Wen et al. [72] used STD-NMR and molecular modelling protocols to probe the binding modes of the glycosidase inhibitors kifunensine and salacinol to Drosophila melanogaster Golgi $\alpha$-mannosidase 11. A comparison of the experimental and theoretical effects of ligand protons in these complexes allowed for the selection of likely ligand binding modes. The results indicated that reliable binding modes of a ligand to a protein in aqueous solution can be obtained with the combined use of STD-NMR spectroscopy and a computational approach.

Becker et al. [73] used ligand-detected ${ }^{1} \mathrm{H}$ NMR experiments, $\mathrm{T}_{1 \mathrm{p}}$-NOESY, diffusion and STD-NMR to distinguish the binding epitopes of propanolol enantiomers 
with alpha(1)-acid glycoprotein. STD epitope mapping provided insights into different orientations of the enantiomers with respect to the alpha(1)-acid glycoprotein binding pocket. It was concluded that ligand-protein interactions can be elucidated by using the combination of several NMR epitope mapping experiments.

Several research groups have proposed the implementation of STD-NMR spectroscopy in chromatographic optimisation studies [74,75]. STD-NMR in combination with molecular dynamics simulations can be used to screen chiral stationary phases for evidence of molecular interactions as a predictor of chiral column selection and to study the mechanism of chemically selective displacement chromatography.

Recently, several improvements have been made in STD-NMR [76,77]. Novel competition STD experiments based on the combination of filtering and isotope labelling schemes have been demonstrated using a mixture containing either unlabelled or ${ }^{13} \mathrm{C}$ labelled D-maltose in the presence of malectin. The signals of the unlabelled ligand are present only in ${ }^{13} \mathrm{C}$-filtered STD spectrum, while the edited experiment shows no signals. However, when using ${ }^{13} \mathrm{C}$ labelled maltose, only the ${ }^{13} \mathrm{C}$-edited experiment produces STD signals. The combination of ${ }^{13} \mathrm{C}$-filtered and ${ }^{13} \mathrm{C}$-edited experiments allows for the separation of the STD signals of the labelled reference ligand from the signals corresponding to the unlabelled hit compounds. Similarly, a novel STD experiment was proposed for group selective saturation of amide protons in ${ }^{15} \mathrm{~N}$ labelled hosts. The pulse sequence of the experiment is based on a train of bilinear rotation decoupling (BIRD) pulses that inverts only protons attached to ${ }^{15} \mathrm{~N}$ and results in saturation of the amide protons. The ${ }^{15} \mathrm{~N}$ GS STD experiment produces clean and artifact-free STD spectra. The feasibility study indicated that ${ }^{15} \mathrm{~N}$ GS STD experiments can be used as the basis for studying intermolecular interactions where the standard STD experiment is difficult to optimise.

TrNOE and molecular dynamics simulations (MD) were used to study the interaction between Serratia marcescens chitinase B (SmChiB) and the dipeptide $\mathrm{N}$ acetyl-Arg- $\left\{N^{\omega}\right.$ - $(N$-methylcarbamoyl $\left.)\right\}-N$-methyl-Phe [78]. The dipeptide was derived from the naturally produced cyclopentapeptide chitinase inhibitor argifin. Molecular dynamics simulations and trNOE experiments indicated that both argifin and the dipeptide bind to ( $\mathrm{SmChiB}$ ) with similar affinities. The less favourable van der Waals and non-polar solvation energies of the dipeptide compared with argifin were compensated for by the improved total electrostatic component.

Several authors have demonstrated the application of NMR methodology based on relaxation rate analysis. Spin-lattice relaxation rate enhancements of ligand protons were used in order to investigate the affinity of epinephrine and isoproterenol for two different systems: fibrinogen and platelets [79]. The affinity index for epinephrine-fibrinogen was three times greater than the isoproterenolfibrinogen affinity index. On the contrary, the affinity indexes for epinephrine- 
platelet and isoproterenol-platelet interactions were similar, indicating that the ligands interact with the receptor with the same strength.

Furthermore, Lerche et al. [80] applied hyperpolarised ${ }^{13} \mathrm{C}$ NMR screening as a competitive assay to characterise the binding of salicylate and ascorbate to human serum albumin. Signal enhancements were achieved using dynamic nuclear polarisation (DNP) ${ }^{13} \mathrm{C}$ NMR spectroscopy. Dynamic nuclear polarisation creates a non-equilibrium state and the enhanced spin polarisation returns to its equilibrium with the relaxation time $T_{1}$. As enhanced polarisation is much faster in macromolecule-bound ligands than in free ligands, relaxation time $T_{1}$ can be used to distinguish free and bound states.

TROSY was applied to investigate ternary systems composed of liver bile acid binding protein, bile acids and membrane mimetic systems, such as anionic liposomes [81]. The absence of the folded protein resonances in the TROSY spectrum of the liposome-protein complex indicated the formation of partially unfolded membrane-bound species. NMR titration of the liposome-protein complex was performed with increasing amounts of bile acids. The signals of the folded protein became more intense at higher bile acid/protein ratios. Hence, the presence of bile acid produced a shift in equilibrium towards the ligand-bound folded protein.

Relaxation rate analysis can be combined with NMR techniques based on monitoring chemical shift perturbations. Shi et al. [82] combined the aforementioned method to investigate the human vinexin SH3 domain. Sitespecific backbone amide $\left({ }^{15} \mathrm{~N}\right){ }^{1} \mathrm{H}$ and side chain ${ }^{19} \mathrm{~F}$ chemical shifts as well as relaxation analysis of $\mathrm{SH} 3$ domains in the absence or presence of a peptide ligand indicated different internal motions due to ligand binding at the three different sites. Similarly, Fenwick et al. [83] performed solution NMR experiments to gain insight into the structure and dynamics of the bilobed ligand-binding core of glutamate receptor 2 in complexes with a set of willardine partial agonists. The regions of the receptor experiencing structural and/or dynamic changes due to interactions with the ligand were identified by main chain amide ${ }^{1} \mathrm{H} /{ }^{15} \mathrm{~N}$ chemical shift deviations. Backbone dynamics on the chemical shift timescale were detected using measurements of ${ }^{15} \mathrm{~N}$ relaxation exchange rates $\left(R_{\mathrm{ex}}\right)$. The NMR results showed that conformational exchange dynamics vary largely with efficacy and the extent of desensitisation.

Bai et al. [84] used global ${ }^{1} \mathrm{H}-{ }^{15} \mathrm{~N}$ HSQC chemical shift perturbations to elucidate the structure of P2-ibuprofen complexes. Two-dimensional ${ }^{1} \mathrm{H}-15 \mathrm{~N}$ HSQC spectra showed that the chemical shift information was communicated from one $\beta$-strand to another, in a "molecular wave" pattern. It was proposed that the ligand induced adjustments in hydrogen bonds stabilising the $\beta$-sheet structure to overcome unfavourable entropic contributions to binding. The information transfer across $\beta$-sheets could be explained by the correlation between the amide nitrogen temperature coefficient and the nitrogen chemical shift perturbation. The correlation between $R$ - and $S$ - ibuprofen temperature coefficients indicated 
that there is little enantiomeric selection for these compounds. The same method was used for the structure determination of 19 distinct ligand-protein complexes in high-throughput ligand affinity screens [85]. The chemical shift perturbation data was used to guide and filter AutoDock calculations using the AutoDockFilter program. The results obtained using chemical shift perturbation experiments were in accordance with the original X-ray structures. Martín-Pastor et al. [86] applied chemical shift perturbation methods to study the interaction of the $\mathrm{GH}$ secretagogue receptor (GHS-R1a) with ghrelin and its des-acyl analogue in living cells. The results showed that ghrelin possesses higher binding affinity than desacylghrelin and that the $n$-octanoic group is involved in the interaction with the (GHS-R1a) receptor. Furthermore, the differences between proton chemical shifts of bound and free ligand were used to elucidate the interaction of the isotopically labelled form of the fully active and chemically defined form of endotoxin, $\mathrm{Kdo}_{2}$-Lipid A, with serum glycoprotein SCD14 [87].

Ligand-protein interactions have been recently investigated by solid-state NMR spectroscopy. Krabben et al. [88] determined the contact area of neurotoxin II from Asian cobra Naja naja oxiana when interacting with membrane-bound nicotinic acetylcholine receptors from Torpedo californica. The comparison of the chemical shift values for the free and receptor-bound toxin indicated that more than $75 \%$ of the assigned ${ }^{13} \mathrm{C}$ resonances did not change within $\pm 0.5 \mathrm{ppm}$. Hence, it was concluded that the observed chemical shift changes were related to large conformational modifications. The strongest chemical shift changes were associated with the residues at the ligand binding interface. Furthermore, Xu et al. [89] assigned uniformly microcrystalline ${ }^{13} \mathrm{C}$ and ${ }^{15} \mathrm{~N}$ enriched yeast triosephosphate isomerase (TIM) using high-resolution solid-state NMR spectroscopy and compared the results with solution-state NMR assignments. An overall agreement between solid-state and solution-state NMR spectra was observed. Some of the observed chemical shift perturbations, as compared to the solution NMR assignment, were related to crystal packing interactions. Furthermore, the binding of a substrate analogue D-glycerol-3-phosphate (D-G3P) to TIM was studied by solid-state NMR for the first time. Site-specific perturbations of the enzyme's chemical shifts upon ligand binding indicated protein conformational changes. Similarly, solid-state ${ }^{2} \mathrm{H}$ NMR spectroscopy was used to investigate the orientation and conformation of retinal within the rhodopsin binding pocket [90]. This approach elucidated the mechanism of rhodopsin activation as a guide for ligand-based drug design.

There are several articles concerning the combined use of NMR spectroscopy and isothermal titration calorimetry. This approach provides a complete thermodynamic characterisation of the investigated system, as was demonstrated with the aminoglycoside antibiotic paromoycin [91]. Furthermore, it was shown that isothermal titration calorimetry in combination with NMR methods was able to elucidate the structure of different enzyme-inhibitor complexes and the dynamics of their interaction [92-94]. 
NMR experiments based on the paramagnetic effects of lanthanide ions can provide unique insight to ligand-protein complex structure. This technique has been used for the structural characterisation of the ternary $30 \mathrm{kDa}$ complex between the lanthanide-labelled N-terminal domain of the epsilon exonuclease subunit from E. coli DNA polymerase III, the theta subunit and thymidine [95].

Although modern ligand-based NMR methods are most widely used to monitor ligand-protein interactions, standard one- and two- dimensional NMR methods are able to structurally characterise the investigated ligand-protein complexes [96-98]. Alcaraz et al. [99] used ${ }^{13}$ C NOESY-HSQC spectra to characterise the adducts of the catalytic domain of matrix metalloprotease-3 (MMP3) with three different non-peptide inhibitors. Similarly, Murail et al. [100] determined the structure of the mouse translocator protein (mTSPO) and the stability of its tertiary folding regarding the presence the of (2-chlorphenyl)- $N$-methyl- $N$-(1-methyl-propyl)-3-isoquinoline carboxamide (PK 11195) ligand using twodimensional NMR methods. The proton chemical shifts and ${ }^{15} \mathrm{~N}-1 \mathrm{H}$ HSQC correlations indicated that PK 11195 binding enhanced the stability of the mTSPO tertiary fold.

As mentioned in the Introduction, ligand-protein interactions can be studied using protein-detected techniques, such as SAR by NMR. Tsantrizos et al. [101] used SAR by NMR to optimise small-molecule hepatitis C virus NS3 protease inhibitors. The observed results have shown that the binding affinity and potency were highly dependent on the ring size, the stereochemistry of each chiral centre and the electrostatic potential of the aromatic substituents.

\subsection{LIGAND-DNA, LIGAND-RNA AND LIGAND MEMBRANE INTERACTIONS}

NMR spectroscopy has also been used to study various interactions of small molecules (ligands) with DNA, RNA and membrane receptors.

Di Micco et al. [102] demonstrated an STD approach based on two parallel sets of STD experiments, in which saturation was elicited by irradiating at a suitable frequency chosen from specific DNA resonances for the characterisation of different DNA ligands. The signal-to-noise ratios of all the protons showing the STD effects were compared with the reference STD spectrum. The results suggested that the ligands making proximate contacts with aromatic base protons received more saturation upon the irradiation of DNA aromatic protons, whereas the external ligands were more affected by irradiation in the deoxyribose region.

Furthermore, ligand-DNA complexes have been studied by diffusion methods. Ramalho et al. [103] utilised a combination of, pulsed field gradient NMR (PFG NMR) spectroscopy, spin-lattice relaxation rate analysis and docking methods to investigate the interaction of 5-nitroimidazole radiosensitisers with duplex DNA. The intensity of radiosensitiser-macromolecule interactions was successfully evaluated using PFG NMR and docking data. Furthermore, the measurements of 
spin-lattice selective relaxation rates represented a powerful tool to investigate the binding affinity of the ligand towards the receptor.

Moreover, novel methods have been proposed for studying ligand-RNA binding.

Kreutz et al. [104] applied ${ }^{19} \mathrm{~F}$ NMR spectroscopy to identify site-specific ligandRNA binding. The approach relied on site-specific labelling of RNA with 2 '-deoxy2 'fluoro(2'-F) nucleosides, thereby replacing the 2'-hydroxy group with a fluorine atom. The downfield shift of fluorine resonance upon ligand binding and a clear decrease of linewidth indicated that RNA has a more rigid structure in the bound state. Furthermore, Foloppe et al. combined NMR spectroscopy and a fluorescence resonance energy transfer (FRET) binding assay to identify aminoacyl-tRNA acceptor site (A-site) ligands [105]. The compounds that showed affinity for the A-site in a FRET-based binding assay were further investigated using NMR spectroscopy. Intermolecular NOEs confirmed the binding of these compounds to RNA.

The study of ligand-membrane interactions using a combination of NMR spectroscopy and other experimental or computational techniques have recently gained particular attention in order to understand metabolic pathways and develop drugs with the desired properties.

Melo et al. [106] studied the interaction between the dengue virus (DV) fusion peptide and different model membranes by fluorescence and NMR spectroscopy. The interaction was strongest in dodecylphosphocholine and anionic phosphatidylcholine/phosphatidylglycerol vesicles, which were the only vesicles fused by the DV fusion peptide. The three-dimensional structure of the DV fusion peptide bound to dodecylphosphocholine micelles and anionic phosphatidylcholine/phosphatidylglycerol vesicles was solved by transferred NOESY (trNOESY) and solution homonuclear NMR spectroscopy. The majority of the trNOE cross-peaks were from the hydrophobic triad, corroborating the dodecylphosphocholinebound structure. Follot et al. [107] used a combination of electron spin resonsance (ESR) and NMR spectroscopy in solution as well as solid-state NMR spectroscopy to investigate the physicochemical properties and membrane interactions of anti-apoptotic 2-(4-fluorophenyl)-3-(pyridine-4-yl)imidazo [1,2-a] pyridine derivatives with different side chain lengths and conformations. The results pointed towards the fact that improved solubility in biological media could be achieved by choosing a six-atom side chain length and that enhancement of membrane interactions/penetration could be achieved by hydroxyl substitutions on the C6 lateral chain. Cabeça et al. [108] applied molecular dynamics (MD) simulations and NMR spectroscopy to examine the interaction of the neutral and protonated species of the local anaesthetic prilocaine (PLC) with phosphatidylcholine (PC) bilayers. The diffusion-ordered 2D NMR spectroscopy (DOSY) experiments showed that neutral PLC has a higher affinity for the liposome environment. Furthermore, STD results revealed that the interaction of prilocaine molecules with the bilayer is $\mathrm{pH}$-dependent. The findings were in accord with MD simulations. 
The progress in NMR spectroscopy for studying interactions of bioactive molecules has been demonstrated in several reviews.

Fielding [109] recently reviewed NMR methods for the determination of proteinligand dissociation constants. A protein and a ligand in thermodynamic equilibrium are characterised by the dissociation constant, $K_{\mathrm{D}}$. In the simplest case of a protein with a single binding site, $K_{\mathrm{D}}$ is defined as:

$$
K_{\mathrm{D}}=\frac{[\mathrm{P}][\mathrm{L}]}{[\mathrm{PL}]}
$$

where $[\mathrm{P}],[\mathrm{L}]$ and $[\mathrm{PL}]$ are the equilibrium concentrations of the protein, ligand and complexed state, respectively. The object of the NMR observation might be the ligand or the protein.

Hence, when viewing the ligand, the NMR experiment has to be able to distinguish between the ligand in the free and bound states, so that [L] and [PL] can be quantified. For a system in fast exchange, the observed NMR response $\left(M_{\mathrm{obs}}\right)$ of a ligand is a molar fraction weighted average of the NMR parameters of the free and bound states:

$$
M_{\mathrm{obs}}=X_{\mathrm{L}(\text { free })} M_{\mathrm{L}(\text { free })}+X_{\mathrm{L}(\text { bound })} M_{\mathrm{L}(\text { bound })}
$$

where $X_{\mathrm{L}(\text { free) }}$ and $X_{\mathrm{L}(\text { bound) }}$ are the molar fractions of the free and bound ligand, and $M_{\mathrm{L}(\text { free) }}$ and $M_{\mathrm{L}(\text { bound) }}$ are the NMR parameters of the ligand in its free and bound states, respectively.

Alternatively, when observing the protein, the NMR experiment has to distinguish between and quantify $[\mathrm{P}]$ and $[\mathrm{PL}]$. For the observation of a protein, the observed NMR response is defined as:

$$
M_{\mathrm{obs}}=X_{\mathrm{P}(\text { free })} M_{\mathrm{P}(\text { free })}+X_{\mathrm{P}(\text { bound })} M_{\mathrm{P}(\text { bound })}
$$

where $X_{\mathrm{P}}$ and $M_{\mathrm{P}}$ are now the molar fraction and the NMR characteristic of the non-bound and occupied protein, respectively.

Kleckner and Foster [110] provided an overview of eight distinct NMR-based methods for studying protein dynamics. The methods are arranged by increasing experimental complexity and the observable timescale of the dynamic exchange $\tau_{\text {ex }}$ :
a) real-time (RT) NMR $\left(\tau_{\text {ex }}>1 \mathrm{~s} ; k_{\text {ex }}<1 \mathrm{~s}^{-1}\right)$,
b) exchange spectroscopy (EXSY) $\left(\tau_{\mathrm{ex}} \approx 10-5000 \mathrm{~ms} ; k_{\mathrm{ex}} \approx 0.2-100 \mathrm{~s}^{-1}\right.$; slow exchange ; $\left.k_{\mathrm{ex}}<<\Delta v\right)$,
c) lineshape analysis ( $\tau_{\mathrm{ex}} \approx 10-100 \mathrm{~ms} ; k_{\mathrm{ex}} \approx 10-100 \mathrm{~s}^{-1}$; slow-intermediate exchange; $k_{\mathrm{ex}} \leq \Delta v$ ),
d) Carr-Purcell Meiboom-Gill relaxation dispersion (CPMG RD) $\left(\tau_{\mathrm{ex}} \approx 0.3-10 \mathrm{~ms} ; k_{\mathrm{ex}} \approx 100-3000 \mathrm{~s}^{-1}\right.$; intermediate-fast exchange $k_{\mathrm{ex}} \approx \Delta \mathrm{v}$ ),
e) Rotating frame relaxation dispersion (RFRD) ( $\tau_{\text {ex }} \approx 20-100 \mu \mathrm{s}$; $k_{\mathrm{ex}} \approx 10000-50000 \mathrm{~s}^{-1}$; intermediate-fast exchange $k_{\mathrm{ex}} \geq \Delta v$ ),
f) Nuclear spin relaxation (NSR) (ps-ns dynamics indirectly probed),
g) Residual dipolar coupling (RDC) (ps-ms dynamics indirectly probed), 
h) Paramagnetic relaxation enhancement (PRE) $\left(\tau_{\mathrm{ex}} \approx 10 \mu \mathrm{s}\right.$; $k_{\mathrm{ex}} \approx 100000 \mathrm{~s}^{-1}$; fast exchange $k_{\mathrm{ex}}>\Delta \Gamma$ ).

Madl et al. [111] discussed the utility and computational approaches of combining solution-state NMR with small-angle X-ray and neutron scattering (SAXS/SANS) experiments for the structural analysis of large protein complexes. The combination of NMR and SAXS/SANS in particular is a valuable approach that complements crystallographic studies for structure determination and the investigation of dynamic properties of multi-domain proteins and their complexes in solution.

Reckel et al. [112] discussed the aim of in-cell NMR spectroscopy. The noninvasive character of in-cell NMR spectroscopy makes it the only biophysical method that enables the direct in-cell observation of conformational changes, post-translational modifications and binding events with high structural resolution in living cells. This unique ability is based on the sensitivity of the chemical shift of an NMR-active nucleus to changes in its chemical environment.

Grzesiek and Sass [113] have reviewed recent advances and applications of solution NMR methods for the characterisation of macromolecular structure and dynamics as well as molecular interactions. The combination of enhanced computational approaches and the in-cell detection of molecules enable a detailed characterisation of folding transitions and intracellular interaction studies.

Blobel et al. [114] have discussed different approaches to monitoring protein interactions by NMR at concentrations close to physiological values in unlabelled species. This review emphasises the role of small molecules and NMR active ${ }^{129} \mathrm{Xe}$ nuclei as reporters in the detection of protein-protein interactions.

Von Itzstein [115] reviewed the latest developments in structure-based inhibitor design for microbial-assisted and mammalian-assisted carbohydrate-recognising proteins. The inhibitors were studied using STD-NMR, crystallography and computational techniques.

Krishna and Jayalakshmi have provided a comprehensive description of complete relaxation and conformational exchange theory adapted for saturation transfer measurements (CORCEMA-ST) [116]. The CORCEMA-ST protocol can be applied to obtain quantitative data from STD-NMR spectra.

CORCEMA-ST is based on the two-state model depicted in Scheme 1. The twostate model involves the ligand and the receptor in their free $\left(\mathrm{L}_{\mathrm{f}}, \mathrm{R}_{\mathrm{f}}\right)$ and bound $\left(\mathrm{R}_{\mathrm{b}}, \mathrm{L}_{\mathrm{b}}\right)$ states. The exchange rate is determined by on- and off-rate constants $\left(k_{\mathrm{L}, \text { on }}, k_{\mathrm{R}, \text { on }}\right.$ and $k_{\mathrm{L}, \mathrm{off}}, k_{\mathrm{R}, \mathrm{off}}$, respectively).

According to the two-state model, the expression of the observable magnetisation experiment is given by:

$$
\mathbf{I}(t)=\mathbf{I}_{\mathbf{o}}+\left[1-\mathrm{e}^{-(\mathbf{R}+\mathbf{K}) t}\right](\mathbf{R}+\mathbf{K})^{-1} \mathbf{Q}
$$




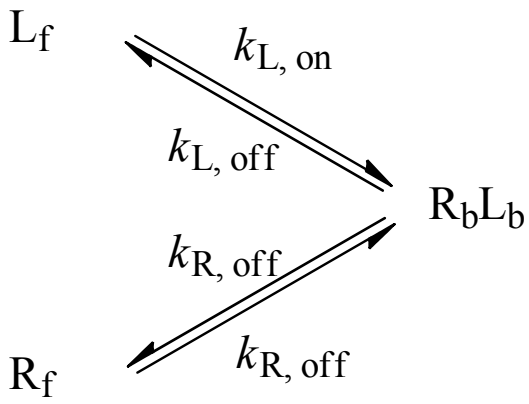

Scheme 1.

where $t$ is the time period in which the protons remain unsaturated. $\mathbf{I}(t)$ is the intensity matrix composed of column matrices $\mathbf{I}_{\mathrm{f}}$ and $\mathbf{I}_{\mathrm{b}}$ representing free and bound protons, respectively. $\mathbf{I}_{\mathrm{o}}$ is the thermal equilibrium matrix, whereas the term $\mathbf{R}+\mathbf{K}$ is the dynamic matrix composed of relaxation rate matrix $\mathbf{R}$ and kinetic matrix $\mathbf{K}$. The matrix $\mathbf{Q}$ is composed of $\mathbf{Q}_{\mathrm{f}}$ and $\mathbf{Q}_{\mathrm{b}}$ submatrices corresponding to the free and bound states, respectively.

A feasibility study was performed utilising a known ligand-protein complex that demonstrated the ability of an STD-NMR intensity-restrained CORCEMA optimisation procedure (SICO) to identify the global minimum conformation for the bound ligand. Furthermore, it was shown that CORCEMA-ST can be successfully utilised in obtaining quantitative structural information on the conformation of ligands within the binding pockets of large proteins.

Goncalves et al. [117] reviewed a recent development in NMR methods for the structural characterisation of $\mathrm{G}$ protein-coupled receptors. This review outlines three key NMR interactions that yield structural information on G proteincoupled receptors:

a) chemical shifts that provide information on the local environment, conformational changes and hydrogen bonding,

b) dipolar couplings used to measure internuclear distances and orientations,

c) quadrupolar couplings that are sensitive to molecular motion.

The investigations of structural changes occurring upon the activation of rhodopsin by a molecular switch mechanism demonstrated the ability of NMR spectroscopy to correlate the structure and function of $\mathrm{G}$ protein-coupled receptors.

Law et al. [118] discussed the role of computational chemistry, NMR spectroscopy and X-ray crystallography in fragment-based drug design (FBDD). The ability of FBDD to optimise ligand fragment properties is illustrated by several ligand-receptor complexes. It was outlined that computational chemistry, in combination with experimental techniques, could have a major impact on the success of FBDD programmes in the future and that it is ready to respond to the challenges that exist in the FBDD field. 
Schanda [119] has provided and excellent review focused on the existing methods for fast multidimensional NMR spectroscopy used for biomolecular studies. The long data acquisition times required for the recording of conventional nD Fourier transform (FT) NMR spectra of biomolecules is very restricting for several reasons: limited stability of biological samples, instrumental costs and the inability to monitor many biologically relevant reactions in real time. These issues can be resolved using different fast multidimensional NMR techniques based on two approaches. The first replaces the regular $(n-1)$-dimensional sampling grid by some other time domain sampling that requires fewer sampling points. Several methods, such as sparse sampling, spectral aliasing and projection NMR, represent this approach. In the second approach, time-domain sampling is replaced by frequency-domain sampling (Hadamard spectroscopy) or a spatial encoding of evolution frequencies (ultrafast NMR spectroscopy).

Fast acquisition methods make NMR ready for use as a powerful tool in the analysis of the structure and dynamics of biological molecules. Hence, fast multidimensional NMR spectroscopy is capable of elucidating phenomena that are out of reach of many other techniques.

Donald and Martin [120] presented an overview of novel algorithms for protein structure determination, automated assignment, characterisation of membrane proteins and protein complexes as well as fold recognition using only unassigned NMR data. The methods presented in this review demonstrate how sparse dipolar couplings can be exploited to resolve the assignment problem, which is a key bottleneck in NMR structural biology.

Schwalbe et al. [25] emphasised the importance of NMR spectroscopy in understanding the structure and dynamics of biomacromolecules in drug discovery. The implementation of novel techniques improved the speed and accuracy of solution- and solid-state NMR spectroscopy. Hence, it was recognised that NMR spectroscopy was capable of determining the structure of mediumsized proteins in native and non-native states as well as characterising ligandreceptor interactions.

\subsection{STRUCTURAL ANALYSIS OF LIGANDS}

The structure of ligands is crucial for their function and interactions with the receptor. Some applications of NMR techniques for ligand structure elucidation are demonstrated by several examples in the following paragraph.

Modarresi-Alam et al. [121] used variable temperature ${ }^{1} \mathrm{H}$ NMR spectroscopy to study restricted S-N rotation in aryl- $N$-(arylsulphonyl)- $N$-(triphenylphosphoranylidene) imidocarbamates. The doublets of the aryl substituents were employed to determine the barriers to rotation at the coalescence temperature. It was shown that a change in the substituents on the oxygen, sulphonyl and nitrogen of the imidoyl moiety had no appreciable influence on the observed barrier to rotation. 
Seneci et al. [122] synthesised and characterised proapoptotic second mitochondria-derived activator of caspases (Smac) mimics and inhibitor of apoptosis protein (IAP) inhibitors. The SAR of this class of inhibitors was elucidated by computational methods and structural studies (NMR spectroscopy, X-ray diffraction). One lead molecule for further in vitro and in vivo evaluation was identified.

Fuchs et al. [123] prepared several heterocyclic analogues of thioflavones based on a Sonogashira-type coupling of $o$-halo(hetero)aroyl chlorides with phenylacetylene. NMR spectroscopic studies provided the structure determination of the prepared compounds.

Zhuang et al. [124] used paramagnetism-based NMR constraints, including fieldinduced RDCs and pseudo-contact shifts (PCSs) for the structure determination of a galectin-3-carbohydrate. The paramagnetic $\mathrm{Dy}^{3+}$ ion was complexed to the peptide, making it possible to observe RDCs as well as PCSs for the protein and the ligand. The determined structure was in agreement with the crystal structure of a galectin-3- $N$-acetyllactosamine complex.

Tiziani et al. [125] applied DOSY to asses possible interactions between daidzein and daidzein soy protein and carotenoids. The results showed that the affinity between daidzein and the soy protein isolate was higher compared to the daidzein dissolved in the same model system. The shifting of the particular proton NMR signals pointed towards two main contributions to the interactions between the phytochemicals and the protein: hydrogen bonding from hydroxyl groups and hydrophobic interactions.

\subsection{STRUCTURAL ANALYSIS OF PEPTIDES AND PROTEINS}

A large number of proteins and peptides serve as ligand receptors. Hence, the elucidation of macromolecular structure and dynamics is crucial for the characterisation of ligand-protein complexes. The recent applications of NMR methods for peptide and protein structure determination are presented in the following section.

Arroyo and Mayo [126] elucidated the structure of angiostatic peptide anginex using NMR structural studies in dodecylphosphocholine micellar environment. Conformational modelling was performed using NOESY data. The results showed that anginex forms a three-stranded anti-parallel $\beta$-sheet conformation in a dodecylphosphocholine micellar solution.

Daly et al. [127] characterised the structure of several cyclotides (cyclo peptides), containing a head-to-tail cyclised peptide backbone and a knotted arrangement of three conserved disulphide bonds. Excellent signal dispersion in NMR spectra makes NMR spectroscopy the most commonly used technique for studying cyclotide structures. The NOESY spectrum of the cyclotide kalata B1 revealed a continuous cycle of sequential cross-peaks, which spanned all 29 amino acids in the sequence and provided evidence for its circular backbone. Furthermore, the 
NMR analysis of kalata B1 and kalata B7 in the presence of dodecylphosphocholine micelles illustrated their binding to the membrane in different orientations.

Furthermore, NMR screening methods were applied to study the structural aspects of allosteric regulation by deuterated functionally active thermostable dimeric Lac repressor proteins [128]. The optimisation of conditions most suitable for lengthy NMR recordings was performed using a high-throughput thermofluor method based on the measurement of the fluorescence intensity of a protein solution at different $\mathrm{pH}$ values, temperatures and salt concentrations.

Yao et al. [129] applied PFG NMR for evaluating the estimates of protein rotational correlation times. The rotational correlation time, $\tau_{c}$, can be expressed as a function of the translation diffusion coefficient, $D_{\mathrm{t}}$ :

$$
\tau_{\mathrm{c}}=\frac{2}{9} \frac{\left(R_{\mathrm{h}}^{\mathrm{ref}} D_{\mathrm{t}}^{\mathrm{ref}}\right)^{2}}{D_{\mathrm{t}}^{3}}
$$

where $R_{\mathrm{h}}{ }^{\text {ref }}$ and $D_{\mathrm{t}}^{\text {ref }}$ are the hydrodynamic radius and diffusion coefficient of the reference molecule, respectively.

The estimates of protein rotational correlation times can be useful if:

a) isotopically labelled material is not available,

b) relaxation rates are difficult to interpret,

c) a full relaxation analysis is difficult because of limited sensitivity.

Kodama et al. [130] used time-sharing NMR measurements as a rapid, effective and unbiased approach for the identification of a protein-protein interface without resonance assignments. Only a single protein ${ }^{15} \mathrm{~N}$ - and ${ }^{13} \mathrm{C}$-labelled sample was required for the analysis. The measurements were performed using a new pulse sequence that allowed simultaneous aromatic ${ }^{1} \mathrm{H}-{ }^{13} \mathrm{C}$ and $\omega_{1}$-TROSY-type backbone ${ }^{1} \mathrm{H}-15 \mathrm{~N}$ correlations, together with single quantum methyl ${ }^{1} \mathrm{H}-13 \mathrm{C}$ correlations.

Sakurai et al. [131] investigated the structure, dynamics and folding of $\beta$ lactoglobulin as well as its interaction with ligands by NMR spectroscopy. The results indicated that $\beta$-lactoglobulin undergoes a conformational change during $\mathrm{pH}$-dependent Tanford transition and ligand binding. The folding mechanism and perturbations due to the introduction of a mutation were explained by a schematic representation using folding funnels.

Buchko et al. [132] studied leucine-rich amelogenin protein (LRAP), a splicevariant isoform of amelogenin using NMR spectroscopy. The main difference in the ${ }^{1} \mathrm{H}-15 \mathrm{~N}$ NMR spectrum of LRAP compared to the full-length amelogenin protein was an additional set of amide resonances for seven non-proline residues, which indicated the presence of two different conformations. The specific changes in molecular dynamics of LRAP upon $\mathrm{NaCl}$ addition, manifested 
by the reduction in intensity and disappearance of ${ }^{1} \mathrm{H}-15 \mathrm{~N}$ HSQC cross-peaks, pointed towards the formation of protein nanospheres.

Moriya et al. [133] determined the protein-protein interface and solvent accessibility upon complex formation between ubiquitin and a C90S mutant of yeast ubiquitin hydrolase 1 (YUH 1) using ${ }^{13} \mathrm{C}$ chemical shift perturbations induced by freely diffusing 4-hydroxy-2,2,6,6-tetramethyl-piperidine-1-oxyl (TEMPOL).

\subsection{MACROLIDE-RIBOSOME INTERACTIONS}

Macrolide antibiotics, such as erythromycin, azithromycin and telitrhomycin (Figure 5.9) are effective therapeutic agents for treating infectious diseases $[134,135]$. They are still the centre of interest of many research groups and pharmaceutical companies and much effort is directed toward the discovery of new macrolide antibiotics by chemical modification of the existing classes of natural derivatives. The goal is to obtain novel therapeutic agents having an improved overall biological profile with a special emphasis on resistant bacterial strains. The continuing emergence of multidrug-resistant bacterial strains imposes a serious threat to the health-care community and intensifies the search for new and more effective agents in order to overcome this problem. Of particular interest are azalides, semisynthetic derivatives of erythromycin A $[134,136]$, which have been found to display a wide antimicrobial spectrum and the ability to concentrate within host cells with high accumulation ratios.

There are two major mechanisms of resistance to macrolides, i.e. target site modifications by methylation that prevent the binding of the antibiotic to the ribosome (encoded by the erm gene) and efflux mechanisms, mediated by the mef (streptococci) and msr (staphylococci) genes. The methylation of ribosomal rRNA bases leads to cross-resistance to macrolides (M), lincosamides (L) and streptogramine B $\left(\mathrm{S}_{\mathrm{B}}\right)$, the so-called $\mathrm{MLS}_{\mathrm{B}}$ phenotype [137].

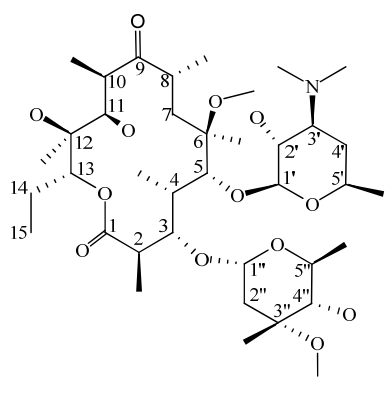

a)

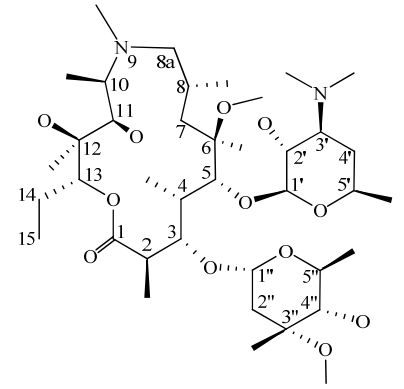

b)

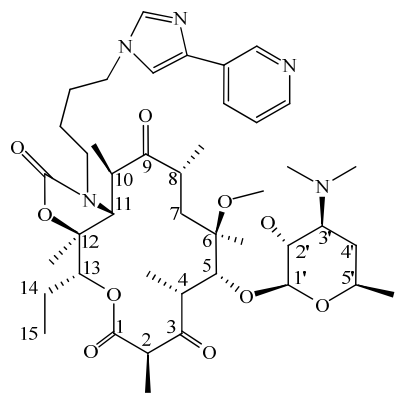

c)

Figure 5.9. Chemical structures of (a) erythromycin, (b) azithromycin and (c) telithromycin

Macrolides exert their activity by interacting with the bacterial $50 \mathrm{~S}$ ribosomal subunit at, or close to, the peptidyl-transferase centre and thus inhibit the growth 
of the nascent peptide chain. As mentioned above, resistance to antibiotics has become a global problem and much effort is now directed toward new and more potent classes of drugs. An effective approach to overcoming this problem is to understand the principles of how these drugs interact with the ribosome [138]. Recently, crystal structures of some ribosome-macrolide complexes [139-141] have shed new light on the binding mechanisms of macrolides to ribosomes and hence provide a good basis for the rational design of new ligands and inhibitors. However, when analysing solid state structures of ribosome-macrolide complexes, one should keep in mind some discrepancies between the structures obtained for the halophilic archeon $H$. Marismortui $[139,141,142]$ and D. radiodurans $[140,143]$. The proposed models differ even though ribosomal 50S subunits of the two bacteria have drug binding sites whose sequences are highly conserved. Furthermore, the crystal structure data obtained so far on complexes of macrolides with ribosomes isolated from clinically non-relevant bacteria do not explain all the effects of macrolides on different pathogenic strains. We believe that steps taken in the process of drug design should also include the elucidation of the solution-state structures of free and bound ligand molecules since the structural features of the complex may not be exactly the same in solution as in the solid state $[142,145]$.

Novak et al. [146-150] have shown that a systematic approach combining NMR and molecular modelling calculations could be applicable to conformational studies of free and bound macrolides and their interactions with ribosomes. Their results [146-150], and those of other groups [151-155], have shown that macrolides adopt two major conformational families, folded-out and folded-in, referring to the outward and inward folding of the ring fragment 3C-5C (Figure 5.10).

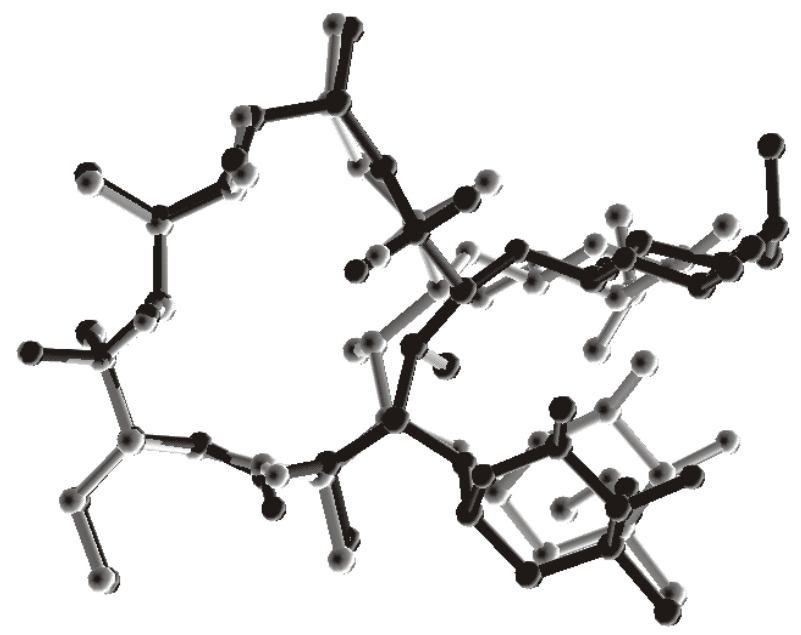

Figure 5.10. Superposition of the two major conformations of azithromycin: folded-in (black) and folded-out (grey)

The authors concluded that the vicinal coupling constants $33_{\mathrm{H} 2 \mathrm{H} 3}$ and NOE protonproton contacts such as $\mathrm{H} 3-\mathrm{H} 11$ and $\mathrm{H} 4-\mathrm{H} 11$ are good indicators of aglycone fold- 
ing. Furthermore, $3_{\mathrm{CH}}$ coupling constants over the glycosidic bonds might provide information about the position and mobility of sugar units, with respect to the lactone ring. Longitudinal relaxation of methyl protons could also be useful to probe motions of methyl groups which reflect aglycone ring folding. Additionally, by applying trNOESY and STD-NMR experiments, it was possible to characterise the interactions of macrolides and ribosomes [146,148].

STD experiments have indicated three common regions closest to the ribosome surface: desosamine sugar, cladinose sugar and the methyl group at position 13 (Figure 5.11).

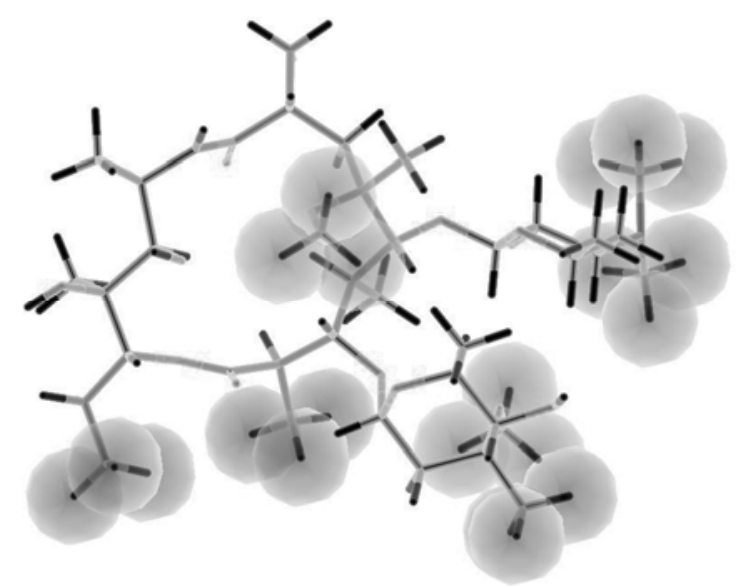

Figure 5.11. STD binding epitopes of a macrolide derivative.

These were also found by X-ray diffraction to be the reactive groups responsible for binding to the ribosome. The bound conformations of the studied macrolides were found to be very similar to those observed in the free state, which were also in agreement with the results obtained by crystallography [142,144]. Furthermore, it was found that the absence of cladinose sugar was the main cause of the inability of decladinosyl macrolides to bind to the ribosome. Recently, more detailed studies using a combination of STD-NMR and in vitro protein inhibition on a series of macrolide compounds have shown that hydrophobic interactions involving cladinose, methyl groups of the lactone ring and the 13-alkyl moiety are a prerequisite for optimal positioning of the desosamine sugar unit. A synergy between the desosamine 3'-dimethylamino and 2'-hydroxyl interactions with the ribosome has been proposed as crucial for successful protein synthesis inhibition. Recently, NMR self-diffusion and solvent paramagnetic relaxation enhancements have been used to determine the interaction strength and localization of a series of macrolide antibiotics with membrane-mimetic micelles. It has been proven that protonated nitrogen atoms play a crucial role in binding to membrane-mimetics [156].

STD-NMR spectroscopy has also been employed to characterise the epitopes of azithromycin, oleandomycin and telithromycin macrolide antibiotic binding to 
bovine serum albumin (BSA) [157]. The structural parts of azithromycin and oleandomycin in intimate contact with bovine serum albumin were found to be similar, while those of telithromycin showed similarities but also some differences. The authors explained these findings by different structural elements of antibiotics that interacted with the protein, especially the alkyl-heteroaryl side chain in telithromycin and cladinose and desosamine sugars in azithromycin and oleandomycin.

\section{Acknowledgement}

This study was supported by the Ministry of Science, Education and Sports of the Republic of Croatia (Project No. 119-1191342-1083).

\section{REFERENCES}

1. M.P. Williamson, T.F.D. Havel, K. Wüthrich. Solution Conformation of Proteinase Inhibitor IIA from Bull Seminal Plasma by ${ }^{1} \mathrm{H}$ Nuclear Magnetic Resonance and Distance Geometry. Journal of Molecular Biology 182(2) (1985) 295-315.

2. K. Wutrich. NMR of Proteins and Nucleic Acid, Wiley, 1986.

3. H. Patzelt, N. Goto, H. Iwai, K. Lundstrom, E. Fernholz. Modern Methods for the Expression of Proteins in Isotopically Enriched Form. In O. Zerbe (Ed.), BioNMR in Drug Research, Wiley-VCH, 2003 pp. 1-38.

4. V. Tugarinov, W.Y. Choy, V.Y. Orekhov, L.E. Ka. Solution NMR-derived Global Fold of a Monomeric 82-kDa Enzyme. Proceedings of the National Academy of Science of the United States of America 102(3) (2005) 622-627.

5. K. Pervushin, R. Riek, G. Wider, K. Wüthrich. Attenuated T2 Relaxation by Mutual Cancellation of Dipole-dipole Coupling and Chemical Shift Anisotropy Indicates an Avenue to NMR Structures of Very Large Biological Macromolecules in Solution. Proceedings of the National Academy of Science of the United States of America 94(23) (1997) 12366-12371.

6. K. Pervushin, R. Riek, G. Wider, K. Wütrich. Transverse Relaxation-Optimized Spectroscopy (TROSY) for NMR Studies of Aromatic Spin Systems in ${ }^{13} \mathrm{C}$-Labeled Proteins. Journal of American Chemical Society 120(25) (1998) 6394-6400.

7. M. Pellechia, P. Sebbel, U. Herrmanns, K. Wüthrich, R. Glockshuber. Pilus chaperone FimC-adhesin FimH interactions mapped by TROSY-NMR. Nature Structural \& Molecular Biology 6(4) (1999) 336-339.

8. R. Bader, M. Lerch, O. Zerbe, NMR of Membrane-Associated Peptides and Proteins. In BioNMR in Drug Research, (Ed: O. Zerbe) Wiley-VCH, 2003 pp. 95-120.

9. C. Fernandez, G. Wider. TROSY in NMR studies of the Structure and Function of Large Biological Macromolecules. Current Opinion in Structural Biology 13(5) (2003) 570-580.

10. A.E. McDermott. Structural and Dynamic Studies of Proteins by Solid-state NMR Spectroscopy: Rapid Movement Forward. Current Opinion in Structural Biology 14(5) (2004) 554-561.

11. J.E. Kay. NMR Studies of Protein Structure and Dynamics. Journal of Magnetic Resonance 173(2) (2005) 193-207. 
12. C.D. Schwieters, J.J. Kuszewski, G. Marius Clore. Using Xplor-NIH for NMR Molecular Structure Determination. Progress in Nuclear Magnetic Resonance Spectroscopy 48(1) (2006) 47-62.

13. M.R. Jensen, P.R.L. Markwick, S. Meier, C. Griesinger, M. Zweckstetter, S. Grzesiek, P. Bernado, M. Blackledge. Quantitative Determination of the Conformational Properties of Partially Folded and Intrinsically Disordered Proteins Using NMR Dipolar Couplings. Structure 17(9) (2009) 1169-1185.

14. M. Coen. A Metabonomic Approach for Mechanistic Exploration of Pre-clinical Toxicology. Toxicology 278(3) (2010) 326-340.

15. J. Griffin. Metabonomics: NMR Spectroscopy and Pattern Recognition Analysis of Body Fluids and Tissues for Characterisation of Xenobiotic Toxicity and Disease Diagnosis. Current Opinion in Chemical Biology 7(5) (2003) 648-654.

16. D. G. Robertson, J. Lindon. Metabonomics in Toxicity Assessment. Taylor \& Francis Group, Boca Raton, 2005.

17. D. Bartos, S. Görög. Recent Advances in the Impurity Profiling of Drugs. Current Pharmaceutical Anaysis 4(4) (2008) 215-230.

18. P. Novak, P. Tepeš, M. Cindrić, M. Ilijaš, S. Dragojevic, K. Mihaljevic. Combined Use of Liquid Chromatography-nuclear Magnetic Resonance Spectroscopy and Liquid Chromatography-mass Spectrometry for the Characterization of an Acarbose Degradation Product. Journal of Chromatography A 1033(2) (2004) 299-303.

19. P. Novak, M. Cindrić, P. Tepeš, S. Dragojević, M. Ilijaš, K. Mihaljević. Identification of Impurities in Acarbose by Using an Integrated Liquid Chromatography-nuclear Magnetic Resonace and Liquid Chromatography-Mass Spectrometry Approach. Journal of Separation Science 28(13) (2005) 1442-1447.

20. P. Novak, P. Tepeš, I. Fistrić, I. Bratoš, V. Gabelica. The Application of LC-NMR and LC-MS for the Separation and Rapid Structure Elucidation of an Unknown in 5aminosalicylic Acid. Journal of Pharmaceutical and Biomedical Analysis 40(5) (2006) 1268-1272.

21. P. Novak, P. Tepeš, M. Ilijaš, I. Fistrić, I. Bratoš, A. Avdagić, V. Gabelica Marković, M. Dumić. Rapid LC-NMR and LC-MS Characterization of an Unknown Component in a Novel Antifungal Drug Icofungipen. Journal of Pharmaceutical and Biomedical Analysis 50(1) (2009) 68-72.

22. B.J. Stockman, C. Dalvit. NMR Screening Techniques in Drug Discovery and Drug Design. Progress in Nuclear Magnetic Resonance Spectroscopy 41 (2002) 187-231.

23. J.W. Peng, J. Moore, N. Abdul-Manan. NMR Experiments for Lead Generation in Drug Discovery. Progress in Nuclear Magnetic Resonance Spectroscopy 44(3) (2004) 225-256.

24. B. Meyer, T. Peters. NMR Spectroscopy Techniques for Screening and Identifying Ligand Binding to Protein Receptors. Angewandte Chemie International Edition 42(8) (2003) 864-890.

25. H. Scwalbe, H.U. Stilz, H. Kessle. NMR Spectroscopy of Biomacromolecules in Drug Discovery and Beyond. ChemBioChem 6(9) (2005) 1475-1478.

26. P.J. Hajduk, J.R. Huth, C. Tse. Predicting Protein Druggability. Drug Discovery Today 10 (23) (2005) 1675-1682.

27. P. Tepeš, P. Novak. Interakcije receptora i liganda pod lupom spektroskopije NMR. Kemija u Industriji 57(4) (2008) 165-173. 
28. L. Fielding. NMR Methods for the Determination of Protein-ligand Dissociation Constants. Progress in Nuclear Magnetic Resonance Spectroscopy 51(4) (2007) 219242.

29. P.J. Hajduk, R.P. Meadows, S.W. Fesik. One-dimensional Relaxation- and Diffusionedited NMR Methods for Screening Compounds that Bind to Macromolecules. Journal of American Chemical Society 119(50) (1997) 12257-12261.

30. S.B. Shuker, P.J. Hajduk, R.P. Meadows, S.W. Fesik. Discovering High-Affinity Ligands for Proteins: SAR by NMR. Science 274(5292) (1996) 1531-1534.

31. P.J. Hajduk, D.J. Augeri, J. Mack, R. Mendoza, J. Yang, S.F. Betz, S.W. Festik. NMRBased Screening of Proteins Containing ${ }^{13} \mathrm{C}$-Labeled Methyl Groups. Journal of American Chemical Society 122(33) (2000) 7898-7904.

32. D. Li, L.A. Levy, S.A. Gabel, M.S. Lebetkin, E.F. Derose, M.J. Wall, E.E. Howell, R.E. London. Interligand Overhauser Effects in Type II Dihydrofolate Reductase. Biochemistry 40(14) (2001) 4242-4252.

33. H. N. Moseley, W. Lee, C.H. Arrowsmith, N. Rama Krishna. Quantitative Determination of Conformational, Dynamic, and Kinetic Parameters of a Ligandprotein/DNA Complex from a Complete Relaxation and Conformational Exchange Matrix Analysis of Intermolecular Transferred NOESY. Biochemistry 36(18) (1997) 5293-5299.

34. M. Mayer, B. Meyer. Characterization of Ligand Binding by Saturation Transfer Difference NMR Spectroscopy. Angewandte Chemie International Edition 38(12) (1999) 1784-1788.

35. M. Mayer, B. Meyer. Group Epitope Mapping by Saturation Transfer Difference NMR To Identify Segments of a Ligand in Direct Contact with a Protein Receptor. Journal of American Chemical Society 123(25) (2001) 6108-6617.

36. M. Vogtherr, T. Peters. Application of NMR Based Binding Assays to Identify Key Hydroxy Groups for Intermolecular Recognition. Journal of American Chemical Society 122(25) (2000) 6093-6099.

37. C. Dalvit, P. Floersheim, M. Zurini, A. Widmer. Use of Organic Solvents and Small Molecules for Locating Binding Sites on Proteins in Solutions. Journal of Biomolecular NMR 14(1) (1999) 23-32.

38. C. Dalvit, P. Pevarello, M. Tatò, M. Veronesi, A. Vulpetti, M. Sundström. Identification of Compounds with Binding Affinity to Proteins via Magnetization Transfer from Bulk Water. Journal of Biomolecular NMR 18(1) (2000) 65-68.

39. I. Bertini, C. Dalvit, J. Gaspard Huber, M. Piccidi. ePHOGSY Experiments on a Paramagnetic Protein: Location of the Catalytic Water Molecule in the Heme Crevice of the Oxidized Form of Horse Heart Cytochrome c. Federationn of European Biochemical Societies Letters 415(1) (1997) 45-48.

40. A. Chen, M.J. Shapiro. NOE Pumping as High-Throughput Method To Determine Compounds with Binding Affinity to Macromolecules by NMR. Journal of American Chemical Society 122(2) (2000) 414-415.

41. J. Orts, C. Griesinger, T. Carolmagno. The INPHARMA Technique for Pharmacophore Mapping: A Theoretical Guide to the Method. Journal of Magnetic Resonance 200 (2009) 64-73.

42. S. Meiboom, D. Gill. Modified Spin-Echo Method for Measuring Nuclear Relaxation Times. Review of Scientific Instruments 29(8) (1958) 688-691. 
43. H.Y. Carr, E.M. Purcell. Effects of Diffusion on Free Precession in Nuclear Magnetic Resonance Experiments. Physical Review 94(3) (1954) 630-638.

44. W. Jahnke, L.B. Perez, C.G. Paris, A. Strauss, G. Feudrich, C.M. Nalin. Second-site NMR Screening with a Spin-Labeled First Ligand. Journal of American Chemical Society 122(30) (2000) 7394-7395.

45. W. Jahnke, S.Rüdisser, M. Eurini. Spin Label Enhanced NMR Screening. Journal of American Chemical Society 123(13) (2001) 3149-3150.

46. G.M. Clore. Exploring Sparsely Populated States of Macromolecules by Diamagnetic and Paramagnetic NMR Relaxation. Protein Science 20(2) (2011) 229-246.

47. P.H.J. Keizers, M. Ubbink. Paramagnetic Tagging for Protein Structure and Dynamics Analysis. Progress in Nuclear Magnetic Resonance Spectroscopy 58(1) (2011) 88-96.

48. T. Madl, W. Bermel, K. Zangger. Use of Relaxation Enhancements in a Paramagnetic Environment for the Structure Determination of Proteins Using NMR Spectroscopy. Angewandte Chemie International Edition 48(44) (2009) 8259-8262.

49. K. Zangger, M. Respondek, C. Göbl, W. Hohlweg, K. Rasmussen, G. Gramp, T. Madl. Positioning of Micelle-bound Peptides by Paramagnetic Relaxation Enhancements. Journal of Physical Chemistry B 113 (2009) 4400-4406.

50. L.H. Lucas, C.K. Larive. Measuring Ligand-protein Binding Using NMR Diffusion Experiments. Concepts of Magnetic Resonance; Part: A 20A(1) (2004) 24-41.

51. C. Dalvit, M. Fasolini, M. Flocco, S. Knapp, P. Pevarello, M. Veronesi. NMR-Based Screening with Competition Water-ligand Observed via Gradient Spectroscopy Experiments: Detection of High-affinity Ligands. Journal of Medicinal Chemistry 45(12) (2002) 2610-2614.

52. C. Dalvit, M. Flocco, S. Knapp, M. Mostardini, R. Perego, B.J. Stockman, M. Veronesi, M. Varasi. High-throughput NMR-based Screening with Competition Binding Experiments. Journal of American Chemical Society 124(26) (2002) 7702-7709.

53. W. Jahnke, P.Floersheim, C. Ostermeier, X. Zhang, R. Hemmig, K. Hurth, D.P. Uzunov. NMR Reporter Screening for the Detection of High-Affinity Ligands. Angewandte Chemie International Edition 41(18) (2002) 3420-3423.

54. A. H. Siriwardena, F. Tian, S. Noble, J.H. Prestegard. A Straightforward NMRSpectroscopy-Based Method for Rapid Library Screening. Angewandte Chemie International Edition 41(18) (2002) 3454-3457.

55. C. Dalvit. Theoretical Analysis of the Competition Ligand-Based NMR Experiments and Selected Applications to Fragment Screening and Binding Constant Measurements. Concepts of Magnetic Resonance; Part: A 32 (5) (2008) 341-372.

56. J. Bandorowicz-Pikula, R. Buchet, F.J. Cañada, M. Clémancey, P. Groves, J. JiménezBarbero, J.M. Lancelin, O. Marcillat, S. Pikula, A. Sekrecka-Belniak, A. Strzelecka-Kiliszek. Characterization of Caged Compounds Binding to Proteins by NMR Spectroscopy. Biochemical Biophysical Research Communications 400(3) (2010) 447-451.

57. A. Blume, A.J. Benie, F. Stolz, R.R. Schmidt, W. Reutter, S. Hinderlich, T. Peters. Characterization of Ligand Binding to the Bifunctional Key Enzyme in the Sialic Acid Biosynthesis by NMR. Journal of Biological Chemistry 279(53) (2004) 5571555721. 
58. G. Bai, B. Feng, J.B. Wang, E. Pozharski, M. Shapiro. Studies on Ligand Binding to Histidine Triad Nucleotide Binding Protein 1. Bioorganic \&Medicinal Chemistry 18(18) (2010) 6756-6762.

59. Z. Ji, Z. Yao, M. Liu. Saturation Transfer Difference Nuclear Magnetic Resonance Study on the Specific Binding of Ligand to Protein. Analytical Biochemistry 385(2) (2009) 380-382.

60. A. Sadeghi-Khomami, M.D. Lumsden, D.L. Jakeman. Glycosidase Inhibition by Macrolide Antibiotics Elucidated by STD-NMR Spectroscopy. Chemistry \& Biology 15(7) (2008) 739-749.

61. M.G. Szczepina, R.B. Zheng, G.C. Completo, T.L. Lowary, B.M. Pinto. STD-NMR Studies Suggest that two Acceptor Substrates for GlfT2, a Bifunctional Galactofuranosyltransferase Required for the Biosynthesis of Mycobacterium tuberculosis Arabinogalactan, Compete for the Same Binding Site. ChemBioChem 10(12) (2009) 2052-2059.

62. A. Blume, M. Fitzen, A.J. Benie, T. Peters. Specificity of Ligand Binding to Yeast Hexokinase PII Studied by STD-NMR. Carbohydrate Research 344(12) (2009) 1567-1574.

63. M. Piazza, L. Yu, A. Teghanemt, T. Gioannini, J. Weiss, F. Peri. Evidence of a Specific Interaction between New Synthetic Antisepsis Agents and CD14. Biochemistry 48(51) (2009) 12337-12344.

64. Y.S. Wang, D. Liu, D.F. Wyss. Competition STD-NMR for the Detection of Highaffinity Ligands and NMR-based Screening. Magnetic Resonance in Chemistry 42(6) (2004) 485-489.

65. L.A. Plesniak, K. Botsch, M. Leibrand, M. Kelly, D. Sem, J.A. Adams, P. Jennings. Transferred NOE and Saturation Transfer Difference NMR Studies of Novobiocin Binding to EnvZ Suggest Binding Mode Similar to DNA Gyrase. Chemical Biology \& Drug Design 71(1) (2008) 28-35.

66. J.Y. Lee, K.W. Jeong, J.U. Lee, D.I. Kang, Y. Kim. Novel E. coli Beta-ketoacyl-acyl Carrier Protein Synthase III Inhibitors as Targeted Antibiotics. Bioorganic \&Medicinal Chemistry 17(4) (2009) 1506-1513.

67. R.S. Houliston, B.C. Jacobs, A.P. Tio-Gillen, J.J. Verschuuren, N.H. Khieu, M. Gilbert, H.C. Jarrel. STD-NMR Used to Elucidate the Fine Binding Specificity of Pathogenic Anti-ganglioside Antibodies Directly in Patient Serum. Biochemistry 48(2) (2009) 220-222.

68. T. Murata, H. Hemmi, M. Nakajima, M. Yoshida, I. Yamaguchi. Epitope Mapping of Gibberellin to the Anti-gibberellin A(4) Monoclonal Antibody by Saturation Transfer Difference NMR spectroscopy. Biochemical Biophysical Research Communications 307(3) (2003) 498-502.

69. F.M. Assadi-Porter, M. Tonelli, E.L. Maillet, J.L. Markley, M. Max. Interactions Between the Human Sweet-sensing T1R2-T1R3 Receptor and Sweeteners Detected by Saturation Transfer Difference NMR Spectroscopy. Biochimica et Biophysica Acta - Biomembranes 1798(2) (2010) 82-86.

70. J.H. Streiff, N. O. Juranic, S.I. Macura, D.O. Warner, K.A. Jones, W.J. Perkins. Saturation Transfer Difference Nuclear Magnetic Resonance Spectroscopy as a Method for Screening Proteins for Anesthetic Binding. Molecular Pharmacology 66(4) (2004) 929-935. 
71. M.A. Macnaughtan, M. Kamar, G. Alvarez-Manilla, A. Venot, J. Glushka, J.M. Pierce, J.H. Prestegard. NMR Structural Characterization of Substrates Bound to Nacetylglucosaminyltransferase V. Journal of Molecular Biology 366(4) (2007) 12661281.

72. X. Wen, Y. Yuan, D.A. Kuntz, D.R. Rose, B.M. Pinto. A Combined STD-NMR/molecular Modeling Protocol for Predicting the Binding Modes of the Glycosidase Inhibitors Kifunensine and Salacinol to Golgi Alpha-mannosidase II. Biochemistry 44(18) (2005) 6729-673.

73. B.A. Becker, C.K. Larive. Probing the Binding of Propranolol Enantiomers to Alpha1-acid Glycoprotein with Ligand-detected NMR Experiments. The Journal of Physical Chemistry B 112(43) (2008) 13581-13587.

74. M.R. Thompson, D.R. McKenzie, J.L. Likos, J.K. Gard. Protein-free Ligand Screening: Simplification of Chiral Chromatographic Development via Novel Adaptation of NMR Screening Methodologies. Magnetic Resonance in Chemistry 47(7) (2009) 541-550.

75. C.J. Morrison, R. Godawat, S.A. McCallum, S. Garde, S.M. Cramer. Mechanistic Studies of Displacer-protein Binding in Chemically Selective Displacement Systems Using NMR and MD Simulations. Biotechnology and Bioengineering 102(5) (2009) 1428-1437.

76. K. Fehér, P. Groves, G. Batta, J. Jiménez-Barbero, C. Muhle-Goll, K.E. Kővér. Competition Saturation Transfer Difference Experiments Improved with Isotope Editing and Filtering Schemes in NMR-based Screening. Journal of American Chemical Society 130(50) (2008) 17148-17153.

77. K.E. Kővér, P. Groves, J. Jiménez-Barbero, G. Batta. Molecular Recognition and Screening Using a ${ }^{15} \mathrm{~N}$ group Selective STD-NMR Method. Journal of American Chemical Society 129(37) (2007) 11579-11582.

78. H. Gouda, T. Sunazuka, T. Hirose, K. Iguchi, N. Yamaotsu, A. Sugawara, Y. Noguchi, Y. Saito, T. Yamamoto, T. Watanabe, K. Shiomi, S. Ōmura, S. Hirono. NMR Spectroscopy and Computational Analysis of Interaction Between Serratia marcescens Chitinase B and a Dipeptide Derived from Natural-product Cyclopentapeptide Chitinase Inhibitor Argifin. Bioorganic \&Medicinal Chemistry 18(16) (2010) 5835-5844.

79. C. Bonechi, S. Martini, C. Rossi. Interaction Study of Bioactive Molecules with Fibrinogen and Human Platelets Determined by ${ }^{1} \mathrm{H}$ NMR Relaxation Experiments. Bioorganic \&Medicinal Chemistry 17(4) (2009) 1630-1635.

80. M.H. Lerche, S. Meier, P.R. Jensen, H. Baumann, B.O. Petersen, M. Karlsson, J.Ø. Duus, J.H. Ardenkjær-Larsen. Study of Molecular Interactions with ${ }^{13} \mathrm{C}$ DNP-NMR. Journal of Magnetic Resonance 203(1) (2010) 52-56.

81. M. Pedò, F. Löhr, M. D’Onofrio, M. Assfalg, V. Dötsch, H. Molinari. The Interplay of Ligand Binding and Quaternary Structure in the Diverse Interactions of Dynein Light Chain LC8. Journal of Molecular Biology 394(5) (2009) 852-863.

82. P. Shi, Z. Xi, H. Wang, C. Shi, Y. Xiong, C. Tian. Site-specific Protein Backbone and Side-chain NMR Chemical Shift and Relaxation Analysis of Human Vinexin SH3 Domain Using a Genetically Encoded 15N/19F-labeled Unnatural Amino Acid. Biochemical Biophysical Research Communications 402(3) (2010) 461-466. 
83. M K. Fenwick, R.E. Oswald. NMR Spectroscopy of the Ligand-binding Core of Ionotropic Glutamate Receptor 2 Bound to 5-substituted Willardiine Partial Agonists. Journal of Molecular Biology 378(3) (2008) 673-685.

84. G. Bai, H. Mo, M .Shapiro. NMR Evaluation of Adipocyte Fatty Acid Binding Protein (aP2) with $R$ - and S-ibuprofen. Bioorganic \&Medicinal Chemistry 16(8) (2008) 4323-4330.

85. J. Stark, R. Powers. Rapid Protein-ligand Costructures Using Chemical Shift Perturbations. Journal of American Chemical Society 130(2) (2008) 535-545.

86. M. Martín-Pastor, A. De Capua, C.J.P. Álvarez, M.D. Díaz-Hernández, J. JiménezBarbero, F.F. Casanueva, Y. Pazos. Interaction between Ghrelin and the Ghrelin Receptor (GHS-R1a), a NMR Study Using Living Cells. Bioorganic \&Medicinal Chemistry 18(4) (2010) 1583-1590.

87. S. Albright, P. Agrawal, N.U. Jain. NMR Spectral Mapping of Lipid A Molecular Patterns Affected by Interaction with the Innate Immune Receptor CD14. Biochemical Biophysical Research Communications 378(4) (2009) 721-726.

88. L. Krabben, B.J. van Rossum, S. Jehle, E. Bocharov, E.N. Lyukmanova, A.A.Schulga, A. Arseniev, F. Hucho, H. Oschkinat. Loop 3 of Short Neurotoxin II is an Additional Interaction Site with Membrane-bound Nicotinic Acetylcholine Receptor as Detected by Solid-state NMR Spectroscopy. Journal of Molecular Biology 390(4) (2009) 662-671.

89. Y. $\mathrm{Xu}$, J. Lorieau, A. E. McDermott. Triosephosphate Isomerase: ${ }^{15} \mathrm{~N}$ and ${ }^{13} \mathrm{C}$ Chemical Shift Assignments and Conformational Change upon Ligand Binding by Magic-angle Spinning Solid-state NMR Spectroscopy. Journal of Molecular Biology 397(1) (2010) 233-248.

90. K. Tanaka, A.V. Struts, S. Krane, N. Fujioka, G.F.J Salgado, K. Martinez-Mayorga, M.F. Brown, K. Nakanishi. Synthesis of $\mathrm{CD}_{3}$-Labeled 11-cis-Retinals and Application to Solid-State Deuterium NMR Spectroscopy of Rhodopsin. Bulletin of the Chemical Society of Japan 80(11) (2007) 2177-2184.

91. C.M. Barbieri, D.S. Pilch. Complete Thermodynamic Characterization of the Multiple Protonation Equilibria of the Aminoglycoside Antibiotic Paromomycin: a Calorimetric and Natural Abundance ${ }^{15}$ N NMR Study. Biophysical Journal 90(4) (2006) 1338-1349.

92. H.W. Huang, S.K. Mohan, C. Yu. The NMR Solution Structure of Human Epidermal Growth Factor (hEGF) at Physiological pH and its Interactions with Suramin. Biochemical Biophysical Research Communications 402(4) (2010) 705-710.

93. C.D. Amero, D.W. Byerly, C.A. McElroy, A. Simmons, M.P. Foster. Ligand-induced Changes in the Structure and Dynamics of Escherichia coli Peptide Deformylase. Biochemistry 48(32) (2009) 7595-7607.

94. K.T. Welch, K.G. Virga, N.A. Whittemore, C. Özen, E. Wright, C.L. Brown, R.E. Lee, E.H. Serpersu. Discovery of Non-carbohydrate Inhibitors of Aminoglycosidemodifying Enzymes. Bioorganic \&Medicinal Chemistry 13(22) (2005) 6252-6263.

95. M. John, G. Pintacuda, A.Y. Park, N.E. Dixon, G. Otting. Structure Determination of Protein-ligand Complexes by Transferred Paramagnetic Shifts. Journal of American Chemical Society 128(39) (2006) 12910-12916.

96. U. Rothweiler, A. Czarna, M. Krajewski, J. Ciombor, C. Kalinski, V. Khazak, G. Ross, N. Skobeleva, L. Weber, T.A. Hodak. Isoquinolin-1-one Inhibitors of the MDM2-p53 Interaction. ChemMedChem 3(7) (2008) 1118-1128. 
97. S. Jin, M.Y. Li, C.Y. Zhu, V. Tran, B.H. Wang. Computer-based de novo Design, Synthesis, and Evaluation of Boronic Acid-based Artificial Receptors for Selective Recognition of Dopamine. ChemBioChem 9(9) (2008)1431-1438.

98. M. Hohwy, L. Spadola, B. Lundquist, P. Hawtin, J. Dahmen, I. Groth-Clausen, E. Nilsson, S. Persdotter, K. von Wachenfeldt, R.H.A. Folmer, K. Edman. Novel Prostaglandin D Synthase Inhibitors Generated by Fragment-based Drug Design. Journal of Medicinal Chemistry 51(7) (2008) 2178-2186.

99. L.A. Alcaraz, L. Banci, I. Bertini, F. Cantini, A. Donaire, L. Gonnelli. Matrix Metalloproteinase-inhibitor Interaction: the Solution Structure of the Catalytic Domain of Human Matrix Metalloproteinase-3 with Different Inhibitors. Journal of Biological Inorganic Chemistry 12(8) (2007) 1197-1206.

100. S. Murail, J.C. Robert, Y.M. Coïc, J.M. Neumann, M.A. Ostuni, Z.X. Yao, V. Papadopoulos, N. Jamin, J.J. Lacapére. Secondary and Tertiary Structures of the Transmembrane Domains of the Translocator Protein TSPO Determined by NMR. Stabilization of the TSPO Tertiary Fold Upon Ligand Binding. Biochimica et Biophysica Acta - Biomembranes 1778(6) (2008) 1375-1381.

101. Y.S. Tsantrizos. The Design of a Potent Inhibitor of the Hepatitis C Virus NS3 Protease: BILN 2061 - From the NMR Tube to the Clinic. Biopolymers 76(4) (2004) 309-323.

102. S. Di Micco, C. Bassarello, G. Bifulco, R. Riccio, L. Gomez-Paloma. DifferentialFrequency Saturation Transfer Difference NMR Spectroscopy Allows the Detection of Different Ligand-DNA Binding Modes. Angewandte Chemie International Edition 45(2) (2006) 224-228.

103. T.C. Ramalho, T.C.C. França, W.A. Cortopassi, A.S. Gonçalves, A.W.S. da Silva, E.F.F. da Cunha. Topology and Dynamics of the Interaction Between 5-nitroimidazole Radiosensitizers and Duplex DNA Studied by a Combination of Docking, Molecular Dynamic Simulations and NMR Spectroscopy. Journal of Molecular Structure 992(1) (2011) 65-71.

104. C. Kreutz, H. Kählig, R. Konrat, R. Micura. A General Approach for the Identification of Site-specific RNA Binders by ${ }^{19} \mathrm{~F}$ NMR Spectroscopy: Proof of Concept. Angewandte Chemie International Edition 45(21) (2006) 3450-3453.

105. N. Foloppe, I.J. Chen, B. Davis, A. Hold, D. Morley, R. Howes. A Structure-Based Strategy to Identify New Molecular Scaffolds Targeting the Bacterial Ribosomal Asite. Bioorganic \&Medicinal Chemistry 12(5) (2004) 935-947.

106. M.N. Melo, F.J.R. Sousa, F.A. Carneiro, M.A.R.B. Castanho, A.P. Valente, F.C.L. Almeida, A.T. Da Poian, R. Mohana-Borges. Interaction of the Dengue Virus Fusion Peptide with Membranes Assessed by NMR: The Essential Role of the Envelope Protein Trp101 for Membrane Fusion. Journal of Molecular Biology 392(3) (2009) 736-746.

107. S. Follot, J.C. Debouzy, D. Crouzier, C. Enguehard-Gueiffier, A. Gueiffier, F. Nachon, B. Lefebvre, F. Fauvelle. Physicochemical Properties and Membrane Interactions of Anti-apoptotic Derivatives 2-(4-fluorophenyl)-3-(pyridin-4-yl)imidazo[1,2a]pyridine Depending on the Hydroxyalkylamino Side Chain Length and Conformation: an NMR and ESR Study. European Journal of Medicinal Chemistry 44(9) (2009) 3509-3518. 
108. L.F. Cabeça, M. Pickholz, E. De Paula, A.J. Marsaioli. Liposome-prilocaine Interaction Mapping Evaluated Through STD-NMR and Molecular Dynamics Simulations. The Journal of Physical Chemistry B 113(8) (2009) 2365-2370.

109. L. Fielding. NMR methods for the Determination of Protein-ligand Dissociation Constants. Progress in Nuclear Magnetic Resonance Spectroscopy 51(4) (2007) 219242.

110. I.R. Kleckner, M.P. Foster. An Introduction to NMR-based Approaches for Measuring Protein Dynamics. Biochimica et Biophysica Acta-Proteins \& Proteomics 1814(8) (2011) 942-968.

111. T. Madl, F. Gabel, M. Sattler. NMR and Small-angle Scattering-based Structural Analysis of Protein Complexes in Solution. Journal of Structural Biology 173(3) (2011) 472-482.

112. S. Reckel, R. Hänsel, F. Löhr, V. Dötsch. In-cell NMR Spectroscopy. Progress in Nuclear Magnetic Resonance Spectroscopy 51(2) (2007) 91-101.

113. S. Grzesiek, H.J. Sass. From Biomolecular Structure to Functional Understanding: New NMR Developments Narrow the Gap. Current Opinion in Structural Biology 19(5) (2009) 585-595.

114. J. Blobel, R. Fayos, J. García, O. Marimon, Y. Pérez, M. Pons. Low-molecular-weight Spies of Protein-protein Interactions. Comptes Rendus Chemie 11(2008) 499-505.

115. M. von Itzstein. Disease-associated Carbohydrate-recognising Proteins and Structure-based Inhibitor Design. Current Opinion in Structural Biology 18(5) (2008) 558-566.

116. M.R. Krishna, V. Jayalakshmi. Complete Relaxation and Conformational Exchange Matrix Analysis of STD-NMR Spectra of Ligand-receptor Complexes. Progress in Nuclear Magnetic Resonance Spectroscopy 49(1) (2006) 1-25.

117. J.A. Goncalves, S. Ahuja, S. Erfani, M. Eilers, S.O. Smith. Structure and Function of G Protein-coupled Receptors Using NMR Spectroscopy. Progress in Nuclear Magnetic Resonance Spectroscopy 57(2) (2010) 159-180.

118. R. Law, O. Barker, J.J. Barker, T. Hesterkamp, R. Godemann, O. Andersen, T. Fryatt, S. Courtney, D. Hallett, M. Whittaker. The Multiple Roles of Computational Chemistry in Fragment-based Drug Design. Journal of Computer-Aided Molecular Design 23(8) (2009) 459-473.

119. P. Schanda. Fast-pulsing Longitudinal Relaxation Optimized Techniques: Enriching the Toolbox of Fast Biomolecular NMR Spectroscopy. Progress in Nuclear Magnetic Resonance Spectroscopy 55(3) (2009) 238-265.

120. B.R. Donald, J. Martin. Automated NMR Assignment and Protein Structure Determination using Sparse Dipolar Coupling Constraints. Progress in Nuclear Magnetic Resonance Spectroscopy 55(2) (2009) 101-127.

121. A.R. Modarresi-Alam, F. Khamooshi, M. Rostamizadeh, H. Keykha, M. Nasrollahzadeh, H.R. Bijanzadeh, E. Kleinpeter. Dynamic ${ }^{1} \mathrm{H}$ NMR Spectroscopic Study of the Restricted SN Rotation in Aryl-N-(arylsulfonyl)-N(triphenylphosphoranylidene)imidocarbamates. Journal of Molecular Structure 841(1) (2007) 61-66.

122. P. Seneci, A. Bianchi, C. Battaglia, L. Belvisi, M. Bolognesi, A. Caprini, F. Cossu, E. de Franco, M. de Matteo, D. Delia, C. Drago, A. Khaled, D. Lecis, L. Manzoni, M. Marizzoni, E. Mastrangelo, M. Milani, I. Motto, E. Moroni, D. Potenza, V. Rizzo, F. 
Servida, E. Turlizzi, M. Varrone, F. Vasile, C. Scolastico. Rational Design, Synthesis and Characterization of Potent, Non-peptidic Smac Mimics/XIAP Inhibitors as Proapoptotic Agents for Cancer Therapy. Bioorganic \&Medicinal Chemistry 17(16) (2009) 5834-5856.

123. F.C. Fuchs, G.A. Eller, W. Holzer. Heterocyclic Analogs of Thioflavones: Synthesis and NMR Spectroscopic Investigations. Molecules 14(9) (2009) 3814-3832

124. T. Zhuang, H.S. Lee, B. Imperiali, J.H. Prestegard. Structure Determination of a Galectin-3-carbohydrate Complex Using Paramagnetism-based NMR Constraints. Protein Science 17(7) (2008) 1220-1231.

125. S. Tiziani, S.J. Schwartz, Y. Vodovotz. Intermolecular Interactions in Phytochemical Model Systems Studied by NMR Diffusion Measurements. Food Chemistry 107(2) (2008) 962-969.

126. M.H. Arroyo, K.H. Mayo. NMR Solution Structure of the Angiostatic Peptide Anginex. Biochimica et Biophysica Acta -Proteins \& Proteomics 1774 (2007) 645-651.

127. N.L. Daly, K.J. Rosengren, D.J. Craik. Discovery, Structure and Biological Activities of Cyclotides. Advanced Drug Delivey Reviews 61(11) (2009) 918-930.

128. J. Romanuka, H. van den Bulke, R. Kaptein, R. Boelens, G.E. Folkers. Novel Strategies to Overcome Expression Problems Encountered with Toxic Proteins: Application to the Production of Lac Repressor Proteins for NMR Studies. Protein Expression Purification 67(2) (2009) 104-112.

129. S. Yao, J.J. Babon, R.S. Norton. Protein Effective Rotational Correlation Times from Translational Self-diffusion Coefficients Measured by PFG-NMR. Biophysical Chemistry 136(2) (2008) 145-151.

130. Y. Kodama, M.L. Reese, N. Shimba, K. Ono, E. Kanamori, V. Dötsch, S. Noguchi, Y. Fukunishi, E. Suzuki, I. Shimada, H. Takahashi. Rapid Identification of Proteinprotein Interfaces for the Construction of a Complex Model Based on Multiple Unassigned Signals by Using Time-sharing NMR Measurements. Journal of Structural Biology 174(3) (2011) 434-442.

131. K. Sakurai, T. Konuma, M. Yagi, Y. Goto. Structural Dynamics and Folding of $\beta$ lactoglobulin Probed by Heteronuclear NMR. Biochimica et Biophysica Acta General Subjects 1790(6) (2009) 527-537.

132. G.W. Buchko, B.J. Tarasevich, J. Roberts, M.L. Snead, W.J. Shaw. A Solution NMR Investigation into the Murine Amelogenin Splice-variant LRAP (Leucine-Rich Amelogenin Protein). Biochimica et Biophysica Acta -Proteins \& Proteomics 1804(9) (2010) 1768-1774.

133. J. Moriya, M. Sakakura, Y. Tokunaga, R.S. Prosser, I. Shimada. An NMR Method for the Determination of Protein Binding Interfaces Using TEMPOL-Induced Chemical Shift Perturbations. Biochimica et Biophysica Acta -General Subjects 1790(10) (2009) 1368-1376.

134. W. Schönfeld, S. Mutak. Azithromycin and novel azalides. In: W. Schönfeld and H. A. Kirst (Eds.), Macrolide Antibiotics, Birkhäuser Verlag, Basel, 2002, pp. 73-95.

135. S. Pal. A Journey Across the Sequential Development of Macrolides and Ketolides Related to Erythromycin. Tetrahedron 62(14) (2006) 3171-3200.

136. S. Mutak. Azalides from Azithromycin to New Azalide Derivatives. Journal of Antibiotics 60(2) (2007) 85-122. 
137. J. Poehlsgaard, S. Douthwaite. Macrolide Antibiotic Interaction and Resistance on the Bacterial Ribosome. Current Opinion in Investigational Drugs 4(2) (2003) 140-148.

138. A.S. Mankin. Macrolide Myths. Current Opinion in Microbiology 11 (2008) 414-421.

139. N. Ban, P. Nissen, J. Hansen, P.B. Moore, T.A. Steiz. The Complete Atomic Structure of the Large Ribosomal Subunit at $2.4 \AA$ Resolution. Science 289(5481) (2000) 905-920.

140. F. Schlünzen, R. Zarivach, J. Harms, A. Bashan, A. Tocilj, R. Albrecht, A. Yonath, F. Franceschi. Structural Basis for the Interaction of Antibiotics with the Peptidyl Transferase Centre in Eubacteria. Nature 413(6858) (2001) 814-821.

141. J.L. Hansen, J.A. Ippolito, N. Ban, P. Nissen, P.B. Moore, T.A. Steitz. The Structures of Four Macrolide Antibiotics Bound to the Large Ribosomal Subunit. Molecular Cell 10(1) (2002) 117-128.

142. D. Tu, G. Blaha, P.B. Moore, T.A. Steitz. Structures of $\mathrm{MLS}_{\mathrm{B}} \mathrm{K}$ Antibiotics Bound to Mutated Large Ribosomal Subunits Provide a Structural Explanation for Resistance. Cell 121(2) (2005) 257-270.

143. F. Schlünzen, J.M. Harms, F. Franceschi, H.A.S. Hansen, H. Bartels, R. Zarivach, A. Yonath. Structural Basis for the Antibiotic Activity of Ketolides and Azalides. Structure 11(3) (2003) 329-338.

144. J.A. Dunkle, L. Xiong, A.S. Mankin, J.H.D. Cate. Structures of the Escherichia coli Ribosome with Antibiotics Bound near the Peptidyl Transferase Center Explain Spectra of Drug Action. Proceedings of the National Academy of Science of the United States of America 107(40) (2010) 17152-17157.

145. M.A. Johnson, B.M. Pinto. Saturation-transfer Difference NMR Studies for the Epitope Mapping of a Carbohydrate-mimetic Peptide Recognized by an Anticarbohydrate Antibody. Bioorganic \&Medicinal Chemistry 12(1) (2004) 295-300.

146. P. Novak, I. Tatić, P. Tepeš, S. Koštrun, J. Barber. Systematic Approach to Understanding Macrolide-ribosome Interactions: NMR and Modeling Studies of Oleandomycin and its Derivatives. The Journal of Physical Chemistry A 110(2) (2006) 572-579.

147. P. Novak, Z. Banić Tomišić, P. Tepeš, G. Lazarevski, J. Plavec, G. Turkalj. Conformational Analysis of Oleandomycin and its 8-methylene-9-oxime Derivative by NMR and Molecular Modeling. Organic \& Biomolecular Chemistry 3(1) (2005) 39-47.

148. P. Novak, J. Barber, A. Čikoš, B. Arsić, J. Plavec, G. Lazarevski, P. Tepeš, N. KošutićHulita. Free and Bound state Structures of 6-O-methyl Homoerythromycins and Epitope Mapping of their Interactions with Ribosomes. Bioorganic \&Medicinal Chemistry 17(16) (2009) 5857-5867.

149. N. Košutić-Hulita, D. Matak-Vinković, M. Vinković, P. Novak, G. Kobrehel, G. Lazarevski. Conformational Behaviour of 11-O-methylazithromycin in the Solid and Solution State. Croatica Chemica Acta 74(2) (2001) 327-341.

150. M.B. Krajačić, M. Dumić, P. Novak, M. Cindrić, S. Koštrun, A. Fajdetić, S. Alihodžić, K. Brajša, N. Kujundžić. Discovery of Novel Ureas and Thioureas of 3-decladinosyl-3hydroxy 15-membered Azalides Active Against Efflux-Mediated Resistant Streptococcus pneumoniae. Bioorganic Medicinal Chemistry Letters 21(2) (2011) 853-856.

151. J.R. Everett, J.W. Tyler. The Conformational Analysis of Erythromycin A. Journal of Chemical Society Perkin Transactions 2(11) (1987) 1659-1667. 
152. W.E. Steinmetz, B.L. Shapiro, J.J. Roberts. The Structure of Erythromycin Enol Ether as a Model for its Activity as a Motilide. Journal of Medicinal Chemistry 45(22) (2002) 4899-4902.

153. G. Lazarevski, M. Vinković, G. Kobrehel, S. Đokić, B. Metelko, D. Vikić-Topić. Conformational Analysis of Azithromycin by Nuclear Magnetic Resonance Spectroscopy and Molecular Modeling. Tetrahedron 49(3) (1993) 721-730.

154. A. Awan, R.J. Brennan, A.C. Regan, J. Barber. The Conformations of the Macrolide Antibiotics Erythromycin A, Azithromycin and Clarithromycin in Aqueous Solution: a ${ }^{1}$ H NMR Study. Journal of Chemical Society Perkin Transactions 2(8) (2000) 16451652.

155. G. Bertho, J. Gharby-Benarous, M. Delaforge, C. Lang, A. Parent, J.P. Girault. Conformational Analysis of Ketolide, Conformations of RU 004 in Solution and Bound to Bacterial Ribosomes. Journal of Medicinal Chemistry 41(18) (1998) 3373-3386.

156. S. Kosol, E. Schrank, M. Bukvić-Krajačić, G. Wagner, H. Meyer, C. Göbl, K. Zangger, P. Novak. Probing the Interactions of Macrolide Antibiotics with Membrane-Mimetics by NMR Spectroscopy. Journal of Medicinal Chemistry, doi:10.1021/jm300647f

157. P. Novak, P. Tepeš, V. Lazić. Epitope Mapping of Macrolide Antibiotics to Bovine Serum Albumin by Saturation Transfer Difference NMR Spectroscopy. Croatica Chemica Acta 80(2) (2007) 211-216. 